ALEA, Lat. Am. J. Probab. Math. Stat. 14, 529-555 (2017)

DOI: $10.30757 /$ ALEA.v14-27

\title{
A supersymmetric approach to martingales related to the vertex-reinforced jump process
}

\author{
Margherita Disertori, Franz Merkl and Silke W.W. Rolles \\ Institute for Applied Mathematics \& Hausdorff Center for Mathematics, \\ University of Bonn, \\ Endenicher Allee 60, D-53115 Bonn, Germany. \\ E-mail address: disertori@iam.uni-bonn.de \\ URL: http://www .iam.uni-bonn.de/users/disertori \\ Mathematical Institute, LMU Munich, \\ Theresienstr. 39, \\ D-80333 Munich, Germany. \\ E-mail address: merkl@math.lmu.de \\ URL: http://www. math.lmu.de/ merkl \\ Technische Universität München, Zentrum Mathematik, Bereich M5, \\ D-85747 Garching bei München, Germany. \\ E-mail address: srolles@ma.tum.de \\ URL: http://www-m5.ma.tum.de/Allgemeines/SilkeRolles
}

\begin{abstract}
Sabot and Zeng have discovered two martingales, one of which played a key role in their investigation of the vertex-reinforced jump process. Starting from the related supersymmetric hyperbolic sigma model, we give an alternative derivation of these two martingales. They turn out to be the first two instances in an infinite hierarchy of martingales, derived from a generating function.
\end{abstract}

\section{Introduction}

Sabot, Tarrès, and Zeng (Sabot et al., 2017+) proved that the vertex-reinforced jump process can be related to a certain random Schrödinger operator. A convenient way to characterize the corresponding random environment $\beta$ is its Laplace transform, investigated in Sabot et al. (2017+) using a matrix decomposition from linear algebra.

Subsequently, Sabot and Zeng (2015) have discovered that a certain field $\psi^{(n)}$ associated to the random Schrödinger operator, on increasing finite pieces (with wired boundary conditions) of an infinite graph exhibits a martingale property. This turns out to be the crucial ingredient to prove, among other things, a characterization

Received by the editors November 23th, 2015; accepted May 10th, 2017.

2010 Mathematics Subject Classification. 60G60 (primary), 60G42, 82B44 (secondary).

Key words and phrases. Nonlinear sigma model, vertex-reinforced jump process, martingale. 
of the recurrence and transience behavior of the vertex-reinforced jump process on an arbitrary locally finite graph and, in a certain parameter regime, a functional central limit theorem for this process on $\mathbb{Z}^{d}$ with $d \geq 3$. Ergodicity with respect to spatial translations of the limit of the mentioned martingale is also one of the key ingredients for Sabot and Zeng's proof of recurrence of linearly edge-reinforced random walk on $\mathbb{Z}^{2}$ with arbitrary constant initial weights.

Sabot and Zeng have also described the (discrete) quadratic variation of the mentioned martingale in terms of a second martingale involving the Green's function of the random Schrödinger operator.

In the present paper, we show that these two martingales are the first two instances of an infinite hierarchy of martingales, described in Corollary 2.7 below. The infinite hierarchy is obtained by expanding a martingale consisting of generating functions; cf. Theorem 2.6.

Our starting point is the supersymmetric hyperbolic sigma model $H^{2 \mid 2}$, invented by Zirnbauer (1991) and investigated by Disertori, Spencer, and Zirnbauer in Disertori et al. (2010). Sabot and Tarrès (2015) showed that this model is related to the mixing measures for both vertex-reinforced jump process and edge-reinforced random walk. Key ingredients in our analysis are the symmetries of $H^{2 \mid 2}$ and a local scaling transformation.

Overview of this article. In Section 2, Zirnbauer's $H^{2 \mid 2}$ model is defined formally and the main results are stated.

In Section 3.1 we introduce the mentioned local scaling transformation of the random field $\left(e^{u}, s\right)$, described by $H^{2 \mid 2}$. In Theorem 3.1 we describe the RadonNikodym derivative of the distribution of the transformed field with respect to the original random field. It allows us also to give a short alternative proof of the Laplace transform of $\beta$ from Sabot et al. (2017+); cf. Corollary 3.2 below. Taking $H^{2 \mid 2}$ as a starting point, the measurability argument required to show the various martingale properties is a little easier than in the random Schrödinger operator approach. This is why we include the argument in Section 3.2.

In Section 4, using Theorem 3.1 and the fact $\mathbb{E}\left[e^{u_{k}}\right]=1$ known from Disertori et al. (2010), we give a short alternative proof for the first martingale from Sabot and Zeng (2015); cf. Theorem 2.5 below. In addition to the local scaling transformation, our proofs in Sections 4 and 6 of the martingale properties use a Kolmogorov consistency discovered by Sabot and Zeng (2015) for the random environment $\beta$.

In Section 5, we first review the symmetries of $H^{2 \mid 2}$ that we need for our proof. These include ordinary Euclidean rotations and a $Q$-supersymmetry introduced in Disertori et al. (2010). Using these (super-)symmetries, we derive Ward identities for certain harmonic functions; see Lemma 5.2. The proof of this lemma is based on two main ingredients. First, the mean value theorem for harmonic functions localizes the average over a circle at its center. Second, a technique from Disertori et al. (2010) localizes the expectation of $Q$-supersymmetric functions at the zero field configuration.

In Section 6, a combination of these Ward identities with the local scaling transformation from Section 3.1 yields a generating martingale. An infinite sequence of martingales is then produced by Taylor expansion.

In Section 7, we use Theorem 2.1, which is also a basic ingredient for the generating martingale, to prove a formula discovered by Letac (2014). 
Finally, in the appendix we explain some geometric background and analyze the precise assumptions for the supersymmetric transformation formula between horospherical and cartesian coordinates to hold.

\section{Definitions and main results}

2.1. Finite graph. Let $\tilde{\mathcal{G}}=(\tilde{V}, \tilde{E})$ be a finite, undirected, connected graph with vertex set $\tilde{V}$ and edge set $\tilde{E}$. The graph is assumed to have no self-loops. We fix a reference vertex $\delta \in \tilde{V}$ and abbreviate $V:=\tilde{V} \backslash\{\delta\}$. We assign positive weights $W_{i j}=W_{j i}>0$ to every undirected edge $(i \sim j) \in \tilde{E}$ and set $W_{i j}=0$ for $i \nsim j$. In particular, $W_{i i}=0$. Let

$$
\begin{aligned}
U_{V} & :=\left\{u=\left(u_{i}\right)_{i \in \tilde{V}} \in \mathbb{R}^{\tilde{V}}: u_{\delta}=0\right\}, \\
\Omega_{V} & :=U_{V} \times U_{V} \\
& =\left\{\left(u=\left(u_{i}\right)_{i \in \tilde{V}}, s=\left(s_{i}\right)_{i \in \tilde{V}}\right) \in \mathbb{R}^{\tilde{V}} \times \mathbb{R}^{\tilde{V}}: u_{\delta}=0, s_{\delta}=0\right\} .
\end{aligned}
$$

For $u \in U_{V}$, we define the (negative) discrete Laplacian $A^{W}(u) \in \mathbb{R}^{\tilde{V} \times \tilde{V}}$ associated to the weights $W_{i j} e^{u_{i}+u_{j}}$ by

$$
A_{i, j}^{W}(u)= \begin{cases}-W_{i j} e^{u_{i}+u_{j}} & \text { for } i \neq j \\ \sum_{k \in \tilde{V}} W_{i k} e^{u_{i}+u_{k}} & \text { for } i=j\end{cases}
$$

Let $A_{V V}^{W}(u)$ denote the submatrix of $A^{W}(u)$ obtained by deleting the $\delta$-th row and column, and $\mathcal{T}$ the set of spanning trees of $\tilde{\mathcal{G}}$.

The $H^{2 \mid 2}$ model on $\tilde{\mathcal{G}}$ is given by a probability measure $\mu^{W}$ on $\Omega_{V}$. Following Disertori et al. (2010) and Disertori and Spencer (2010), it can be written in the two following equivalent ways:

$$
\begin{aligned}
& \mu^{W}(d u d s) \\
= & \prod_{(i \sim j) \in \tilde{E}} e^{-W_{i j}\left[\cosh \left(u_{i}-u_{j}\right)+\frac{1}{2}\left(s_{i}-s_{j}\right)^{2} e^{u_{i}+u_{j}}-1\right]} \sum_{T \in \mathcal{T}} \prod_{(i \sim j) \in T} W_{i j} e^{u_{i}+u_{j}} \prod_{i \in V} e^{-u_{i}} \frac{d u_{i} d s_{i}}{2 \pi} \\
= & e^{-\frac{1}{2} s^{t} A^{W}(u) s} \operatorname{det} A_{V V}^{W}(u) \prod_{(i \sim j) \in \tilde{E}} e^{-W_{i j}\left[\cosh \left(u_{i}-u_{j}\right)-1\right]} \prod_{i \in V} e^{-u_{i}} \frac{d u_{i} d s_{i}}{2 \pi}
\end{aligned}
$$

with $d u_{i}$ and $d s_{i}$ denoting the Lebesgue measure on $\mathbb{R}$. Recall that $s_{\delta}=0$; hence we need only the submatrix $A_{V V}^{W}$ to evaluate the quadratic form $s^{t} A^{W} s$. Because the graph $\tilde{\mathcal{G}}$ is connected, this quadratic form with the constraint $s_{\delta}=0$ is positive definite. In particular, the matrix $A_{V V}^{W}$ is invertible.

We define the Green's function $\hat{G}=\hat{G}^{V}=\hat{G}^{V, W}: U_{V} \rightarrow \mathbb{R}^{\tilde{V} \times \tilde{V}}$ by

$$
\hat{G}_{i j}(u)= \begin{cases}e^{u_{i}}\left(A_{V V}^{W}(u)^{-1}\right)_{i j} e^{u_{j}} & \text { for } i, j \in V, \\ 0 & \text { for } i=\delta \text { or } j=\delta .\end{cases}
$$

Note that this definition is equivalent to the representation of $\hat{G}$ given in formula (4.4) in Sabot and Zeng (2015). Furthermore, we introduce the random vector $\beta^{V, W}(u)=\left(\beta_{i}^{V, W}(u)\right)_{i \in V}$ by

$$
\beta_{i}^{V, W}(u)=\frac{1}{2} \sum_{j \in \tilde{V}: j \sim i} W_{i j} e^{u_{j}-u_{i}} .
$$


When there is no risk of confusion we use the notation $\beta, \beta^{V}$, or $\beta^{W}$ (according to which dependence we want to stress) instead of $\beta^{V, W}$.

We denote by $\mathbb{E}_{\mu^{W}}$ the expectation with respect to $\mu^{W}$ and by $\langle a, b\rangle=\sum_{i \in I} a_{i} b_{i}$ the Euclidean scalar product, where $I=V$ or $I=\tilde{V}$ depending on the context. We will also need the following space:

$$
\Lambda_{V}:=\left\{\lambda=\left(\lambda_{i}\right)_{i \in \tilde{V}} \in(-1, \infty)^{\tilde{V}}: \lambda_{\delta}=0\right\} .
$$

For $\lambda \in \Lambda_{V}$, we denote by $\lambda_{V}$ its restriction to $V$. Real functions of $\lambda$, like $\sqrt{1+\lambda}$, are understood componentwise. We abbreviate $e_{\tilde{V}}^{u}=\left(e^{u_{i}}\right)_{i \in \tilde{V}}$.

The main result of this section is the following generalization of the Laplace transform of $\beta=\left(\beta_{i}\right)_{i \in V}$.

Theorem 2.1. For all $\theta \in(-\infty, 0]^{\tilde{V}}$ and all $\lambda \in \Lambda_{V}$, one has

$$
\mathbb{E}_{\mu^{W}}\left[e^{\left\langle\theta, e_{\tilde{V}}^{u}\right\rangle-\frac{1}{2}\langle\theta, \hat{G}(u) \theta\rangle} e^{-\left\langle\lambda_{V}, \beta(u)\right\rangle}\right]=\mathcal{L}^{W}(\lambda) e^{\langle\theta, \sqrt{1+\lambda}\rangle},
$$

where

$$
\mathcal{L}^{W}(\lambda)=\prod_{(i \sim j) \in \tilde{E}} e^{W_{i j}\left(1-\sqrt{1+\lambda_{i}} \sqrt{1+\lambda_{j}}\right)} \prod_{i \in V} \frac{1}{\sqrt{1+\lambda_{i}}} .
$$

The proof is done in Section 6. For $\theta=0$ equation (2.8) gives indeed the Laplace transform $\mathcal{L}^{W}(\lambda)$ of $\beta$. This special case appeared first in Proposition 1 of Sabot et al. (2017+) in the context of a random Schrödinger operator approach. The equivalence of this approach to $H^{2 \mid 2}$ is shown in Corollary 2 of Sabot et al. $(2017+)$. In particular the joint distribution of the $\beta_{i}$ 's is a marginal of their $\nu^{W, 1}$. Using a local scaling transformation, we will give an alternative derivation of the Laplace transform $\mathcal{L}^{W}(\lambda)$ in Corollary 3.2.

For any vector $b=\left(b_{i}\right)_{i \in V} \in \mathbb{R}^{V}$, we define

$$
\left(H_{b}\right)_{i j}=2 b_{i} \delta_{i j}-W_{i j}, \quad i, j \in V \text {. }
$$

In particular,

$$
\left(H_{\beta(u)}\right)_{i j}=2 \beta_{i}(u) \delta_{i j}-W_{i j}=e^{-u_{i}} A_{i j}^{W}(u) e^{-u_{j}}, \quad i, j \in V,
$$

where the last equality follows from the definition (2.6) of $\beta$ and (2.3) of $A^{W}$. Since $A_{V V}^{W}$ is positive definite, the matrix $H_{\beta(u)}$ is invertible. Hence,

$$
H_{\beta(u)}^{-1}=\hat{G}_{V V}^{V, W}(u)
$$

is the restriction of $\hat{G}^{V, W}(u)$, defined in (2.5), to $V \times V$.

The following result is a consequence of Theorem 2.1. ${ }^{1}$

\footnotetext{
${ }^{1}$ Xiaolin Zeng has told us that Gérard Letac has proved formula (2.13) with an inductive approach using linear algebraic methods. Unfortunately, this proof is not published. We were wondering whether Theorem 2.1 is related to Letac's formula. Xiaolin Zeng and Christophe Sabot have answered this question in the affirmative. Christophe Sabot (private communication) showed that Theorem 2.1 can be derived from Letac's formula. Here, we go in the opposite direction and deduce Letac's formula from Theorem 2.1. After the first version of this paper was written, Pierre Tarrès sent us a copy of Letac's manuscript (Letac, 2014).
} 
Corollary 2.2 (Letac's formula, Theorem 2.1 in Letac, 2014). For all $\phi, \theta \in$ $(0, \infty)^{V}$, one has

$$
\int_{\left\{b \in \mathbb{R}^{V}: H_{b}>0\right\}} \frac{e^{-\frac{1}{2}\left(\left\langle\phi, H_{b} \phi\right\rangle+\left\langle\theta, H_{b}^{-1} \theta\right\rangle\right)}}{\sqrt{\operatorname{det} H_{b}}} d b=\left(\frac{\pi}{2}\right)^{\frac{|V|}{2}} \frac{e^{-\langle\phi, \theta\rangle}}{\prod_{i \in V} \phi_{i}},
$$

where the notation $H_{b}>0$ means that $H_{b}$ is positive definite.

To construct the martingale hierarchy, we will need to write $u$ as a function of $\beta$, i.e. to invert the relation (2.6). This is formalized in the following lemma. Recall that $\beta^{V}=\left(\beta_{i}^{V}\right)_{i \in V}$.

Lemma 2.3 (Measurability, Sabot, Tarrès, and Zeng in Sabot et al., 2017+).

We introduce a measurable function $f_{V}^{W}: \mathbb{R}^{V} \rightarrow \mathbb{R}^{\tilde{V}}$ as follows. Given $b \in \mathbb{R}^{V}$ such that $H_{b}$ is invertible, we set

$$
f_{V}^{W}(b)_{\delta}=0 \quad \text { and } \quad f_{V}^{W}(b)_{i}=\log \left[\sum_{j \in V}\left(H_{b}^{-1}\right)_{i j} W_{j \delta}\right] \text { for } i \in V .
$$

In all other cases, we set $f_{V}^{W}(b)=0$. In particular, we have

$$
\left(u_{i}\right)_{i \in \tilde{V}}=f_{V}^{W}\left(\beta^{V}\right) .
$$

Consequently, the random vector $\left(u_{i}\right)_{i \in \tilde{V}}$ is measurable with respect to the $\sigma$-field $\sigma\left(\beta^{V}\right)$.

Given the equivalence of $H^{2 \mid 2}$ and a random Schrödinger description mentioned above, this lemma follows from Proposition 2 in Sabot et al. $(2017+)$. However, since our starting point is $H^{2 \mid 2}$ rather than random Schrödinger operators, we include the proof in Section 3.2 below.

2.2. Infinite graph. Let $\mathcal{G}_{\infty}=\left(V_{\infty}, E_{\infty}\right)$ be an infinite locally finite connected undirected graph without direct loops. We approximate $\mathcal{G}_{\infty}$ by finite subgraphs $\mathcal{G}_{n}=\left(V_{n}, E_{n}\right)$ such that $V_{n} \uparrow V_{\infty}$ and $E_{n}=\left\{(i \sim j) \in E_{\infty}: i, j \in V_{n}\right\}$. Given $n$, we obtain a new finite graph $\tilde{\mathcal{G}}_{n}=\left(\tilde{V}_{n}, \tilde{E}_{n}\right)$ from $\mathcal{G}_{\infty}$ by collapsing all vertices in $V_{\infty} \backslash V_{n}$ to a single vertex $\delta_{n}$. Thus, $\tilde{V}_{n}=V_{n} \cup\left\{\delta_{n}\right\}$ and

$$
\tilde{E}_{n}=E_{n} \cup\left\{\left(i \sim \delta_{n}\right): i \in V_{n} \text { and } \exists j \in V_{\infty} \backslash V_{n} \text { such that }(i \sim j) \in E_{\infty}\right\} .
$$

In other words, $\tilde{\mathcal{G}}_{n}$ is obtained from $\mathcal{G}_{n}$ introducing wired boundary conditions. As in Section 2.1, we assign positive weights $W_{i j}=W_{j i}>0$ to every undirected edge $(i \sim j) \in E_{\infty}$ and we set $W_{i j}=0$ for $i \nsim j$. For $i, j \in \tilde{V}_{n}$, we define the weight $W_{i j}^{(n)}=W_{j i}^{(n)}$ as follows:

$$
\begin{aligned}
& W_{i j}^{(n)}=W_{i j} \quad \text { if } i \in V_{n} \text { and } j \in V_{n}, \\
& W_{i \delta_{n}}^{(n)}=W_{\delta_{n} i}^{(n)}=\sum_{j \in V_{\infty} \backslash V_{n}} W_{i j} \quad \text { for } i \in V_{n}, \quad \text { and } \quad W_{\delta_{n} \delta_{n}}^{(n)}=0 .
\end{aligned}
$$

In particular, $W_{i j}^{(n)}>0$ if and only if $(i \sim j) \in \tilde{E}_{n}$.

Let $\mu_{n}^{W}$ denote the $H^{2 \mid 2}$-measure defined in (2.4) for the graph $\tilde{\mathcal{G}}_{n}$ and edge weights $W_{i j}^{(n)}$. The following observation was made by Sabot and Zeng (2015). To make the presentation self-contained, we will repeat their argument in Section 4. 
Lemma 2.4. (Kolmogorov consistency, Sabot and Zeng, 2015) For $n \in \mathbb{N}$, the Laplace transform $\mathcal{L}_{n}^{W}(\lambda)=\mathbb{E}_{\mu_{n}^{W}}\left[e^{-\left\langle\lambda_{V_{n}}, \beta^{V_{n}}\right\rangle}\right]$ of $\beta^{V_{n}}=\left(\beta_{i}\right)_{i \in V_{n}}$ satisfies the consistency relation

$$
\mathcal{L}_{n}^{W}(\lambda)=\mathcal{L}_{n+1}^{W}(\lambda)
$$

for all $\lambda \in \Lambda_{V_{n+1}}$ with $\lambda_{i}=0$ for all $i \in \tilde{V}_{n+1} \backslash V_{n}$. In particular, the law of $\beta^{V_{n}}$ with respect to $\mu_{n}^{W}$ agrees with the law of $\left.\beta^{V_{n+1}}\right|_{V_{n}}$ with respect to $\mu_{n+1}^{W}$.

Consequently, as worked out in Sabot and Zeng (2015), Kolmogorov's extension theorem yields the existence of a coupling $\left(\boldsymbol{\beta}_{i}\right)_{i \in V_{\infty}}$ on a probability space $\left(\Omega_{\infty}, \mathcal{F}_{\infty}, \mu_{\infty}^{W}\right)$ such that for any $n \in \mathbb{N}$ the laws of the random vectors

$$
\boldsymbol{\beta}^{(n)}=\left(\boldsymbol{\beta}_{i}: \Omega_{\infty} \rightarrow \mathbb{R}\right)_{i \in V_{n}}
$$

with respect to $\mu_{\infty}^{W}$ and $\beta^{V_{n}}: \Omega_{V_{n}} \rightarrow \mathbb{R}^{V_{n}}$ with respect to $\mu_{n}^{W}$ coincide; recall the definition (2.2) of $\Omega_{V_{n}}$. Following Sabot and Zeng (2015), we define the $\sigma$-field

$$
\mathcal{F}_{n}=\sigma\left(\beta^{(n)}\right) \subseteq \mathcal{F}_{\infty} .
$$

Using the function $f_{V}^{W}$ from Lemma 2.3, we define

$$
u^{(n)}=\left(u_{i}^{(n)}\right)_{i \in \tilde{V}_{n}}=f_{V_{n}}^{W}\left(\boldsymbol{\beta}^{(n)}\right) .
$$

In particular, for all $n$, the law of $u^{(n)}$ with respect to $\mu_{\infty}^{W}$ coincides with the law of $u=\left(u_{i}\right)_{i \in \tilde{V}_{n}}$ with respect to $\mu_{n}^{W}$. We also define

$$
u_{i}^{(n)}=u_{\delta_{n}}^{(n)}=0 \quad \text { for } i \in V_{\infty} \backslash V_{n} .
$$

In Section 4, we present an alternative short proof of the following first martingale from Proposition 9 in Sabot and Zeng (2015).

Theorem 2.5 (Martingale $e^{u}$, Sabot and Zeng, 2015). For any $k \in V_{\infty}$, the process $\left(e^{u_{k}^{(n)}}\right)_{n \in \mathbb{N}}$ is a martingale with respect to the filtration $\left(\mathcal{F}_{n}\right)_{n \in \mathbb{N}}$ :

$$
\mathbb{E}_{\mu_{\infty}^{W}}\left[e^{u_{k}^{(n+1)}} \mid \mathcal{F}_{n}\right]=e^{u_{k}^{(n)}}, \quad \forall k \in V_{\infty} .
$$

This martingale will now be generalized. Recall the definition (2.5) of the Green's function $\hat{G}$. We denote by $\hat{G}^{(n)}=\hat{G}^{V_{n}}\left(u^{(n)}\right)$ the Green's function (on the graph $\tilde{\mathcal{G}}_{n}$ ) obtained by replacing $u$ by $u^{(n)}$. Let $(-\infty, 0]^{\left(V_{\infty}\right)}$ denote the set of all $\theta \in(-\infty, 0]^{V_{\infty}}$ having only finitely many non-zero entries. For these $\theta$ and $n \in \mathbb{N}$, we define $\theta^{(n)} \in(-\infty, 0]^{\tilde{V}^{(n)}}$ by

$$
\theta_{i}^{(n)}=\theta_{i} \quad \text { for } i \in V_{n} \quad \text { and } \quad \theta_{\delta_{n}}^{(n)}=\sum_{j \in V_{\infty} \backslash V_{n}} \theta_{j} .
$$

Theorem 2.6 (Generating martingale). For all $\theta \in(-\infty, 0]^{\left(V_{\infty}\right)}$,

$$
M^{(n)}(\theta)=e^{\left\langle\theta^{(n)}, e^{u^{(n)}}\right\rangle-\frac{1}{2}\left\langle\theta^{(n)}, \hat{G}^{(n)} \theta^{(n)}\right\rangle}, \quad n \in \mathbb{N},
$$

is a martingale with respect to the filtration $\left(\mathcal{F}_{n}\right)_{n \in \mathbb{N}}$ defined in (2.21).

The martingale $M^{(n)}(\theta)$ is the generating function for an infinite hierarchy of martingales. The first two martingales (2.27) and (2.28) in this hierarchy are the martingales discovered by Sabot and Zeng; see Proposition 9 in Sabot and Zeng (2015).

In the following, we use the notation $\hat{G}_{k l}^{(n)}=\hat{G}_{l k}^{(n)}=0$ for $k \in V_{\infty} \backslash V_{n}, l \in V_{\infty}$. 
Corollary 2.7 (Hierarchy of martingales). For any $j, k, l \in V_{\infty}$,

$$
\begin{aligned}
& M_{j}^{(n)}=e^{u_{j}^{(n)}}, \quad n \in \mathbb{N}, \\
& M_{j, k}^{(n)}=e^{u_{j}^{(n)}+u_{k}^{(n)}}-\hat{G}_{j k}^{(n)}, \quad n \in \mathbb{N}, \quad \text { and } \\
& M_{j, k, l}^{(n)}=e^{u_{j}^{(n)}+u_{k}^{(n)}+u_{l}^{(n)}}-e^{u_{j}^{(n)}} \hat{G}_{k l}^{(n)}-e^{u_{k}^{(n)}} \hat{G}_{j l}^{(n)}-e^{u_{l}^{(n)}} \hat{G}_{j k}^{(n)}, \quad n \in \mathbb{N},
\end{aligned}
$$

are martingales with respect to the filtration $\left(\mathcal{F}_{n}\right)_{n \in \mathbb{N}}$. More generally, for any $m \in \mathbb{N}$ and any $i_{1}, \ldots, i_{m} \in V_{\infty}$,

$$
M_{i_{1}, \ldots, i_{m}}^{(n)}=\sum_{\substack{I \subseteq\{1, \ldots, m\} \\|I| \text { even }}} \sum_{\mathcal{I} \in \mathcal{P}_{2}(I)}(-1)^{|I| / 2} \prod_{k \in\{1, \ldots, m\} \backslash I} e^{u_{i_{k}}^{(n)}} \prod_{\{k, l\} \in \mathcal{I}} \hat{G}_{i_{k} i_{l}}^{(n)}, \quad n \in \mathbb{N}
$$

are martingales with respect to the same filtration, where $\mathcal{P}_{2}(I)$ denotes the set of all partitions of $I$ in sets of size 2.

Note that the case $I=\emptyset$ corresponds to $\mathcal{P}_{2}(I)=\{\emptyset\}$ and the term $\prod_{k=1}^{m} e^{u_{i_{k}}^{(n)}}$ in the right-hand side of (2.30).

\section{Some tools}

3.1. Local scaling transformation. Fix $\lambda \in \Lambda_{V}$. We define the local shift

$$
S_{\lambda}: \Omega_{V} \rightarrow \Omega_{V}, \quad(\tilde{u}, s) \mapsto(u, s) \quad \text { with } u_{i}=\tilde{u}_{i}+\log \sqrt{1+\lambda_{i}} \text { for all } i \in \tilde{V} .
$$

In particular, $S_{\lambda}$ leaves the $s$-variables unchanged and $\tilde{u}_{\delta}=u_{\delta}=0$. We also introduce the rescaled weights

$$
W_{i j}^{\lambda}=W_{j i}^{\lambda}=\sqrt{1+\lambda_{i}} \sqrt{1+\lambda_{j}} W_{i j} .
$$

The following theorem describes a key property of the local scaling transformation $S_{\lambda}$. Note that similar computations appear also in the proof of Theorem 2(ii) on page 2365 in Sabot and Tarrès (2015).

Theorem 3.1 (Measure transformation). For all $\lambda \in \Lambda_{V}$, the image of $\mu^{W^{\lambda}}$ with respect to the transformation $S_{\lambda}$ is given by

$$
\begin{aligned}
S_{\lambda} \mu^{W^{\lambda}}(d u d s) & =\prod_{(i \sim j) \in \tilde{E}} e^{W_{i j}^{\lambda}-W_{i j}} \cdot \prod_{j \in V} \sqrt{1+\lambda_{j}} e^{-\lambda_{j} \beta_{j}^{W}(u)} \mu^{W}(d u d s) \\
& =\prod_{(i \sim j) \in \tilde{E}} e^{W_{i j}\left(\sqrt{1+\lambda_{i}} \sqrt{1+\lambda_{j}}-1\right)} \cdot \prod_{j \in V} \sqrt{1+\lambda_{j}} e^{-\lambda_{j} \beta_{j}^{W}(u)} \mu^{W}(d u d s) .
\end{aligned}
$$

Remark. Note that (3.3) gives the general formula for $S_{\lambda} \mu^{W}$ :

$$
S_{\lambda} \mu^{W}(d u d s)=\prod_{(i \sim j) \in \tilde{E}} e^{W_{i j}-W_{i j}^{\lambda^{\prime}}} \cdot \prod_{j \in V} \frac{1}{\sqrt{1+\lambda_{j}^{\prime}}} e^{\frac{\lambda_{j}^{\prime}}{1+\lambda_{j}^{\prime}} \beta_{j}^{W^{\lambda^{\prime}}}(u)} \mu^{W^{\lambda^{\prime}}}(d u d s),
$$

where $\lambda_{j}^{\prime}=-\lambda_{j} /\left(1+\lambda_{j}\right)$ so that $\sqrt{1+\lambda_{j}} \sqrt{1+\lambda_{j}^{\prime}}=1$ and the weights satisfy $W_{i j}^{\lambda^{\prime}}=W_{i j} /\left(\sqrt{1+\lambda_{i}} \sqrt{1+\lambda_{j}}\right)$. 
Proof of Theorem 3.1: Using the definition (2.4) of $\mu^{W}$, we find

$$
\begin{aligned}
& \mu^{W^{\lambda}}(d \tilde{u} d s) \\
&= e^{-\frac{1}{2} s^{t} A^{W^{\lambda}}(\tilde{u}) s} \operatorname{det} A_{V V}^{W^{\lambda}}(\tilde{u}) \\
& \prod_{(i \sim j) \in \tilde{E}} e^{-W_{i j}^{\lambda}\left[\cosh \left(\tilde{u}_{i}-\tilde{u}_{j}\right)-1\right]} \prod_{i \in V} e^{-\tilde{u}_{i}} \frac{d \tilde{u}_{i} d s_{i}}{2 \pi} .
\end{aligned}
$$

Fix $(u, s) \in \Omega_{V}$ and set $(\tilde{u}, s)=S_{\lambda}^{-1}(u, s)=\left(\left(u_{i}-\log \sqrt{1+\lambda_{i}}\right)_{i \in \tilde{V}}, s\right) \in \Omega_{V}$ as in (3.1). From $W_{i j}^{\lambda} e^{\tilde{u}_{i}+\tilde{u}_{j}}=W_{i j} e^{u_{i}+u_{j}}$ for $i, j \in \tilde{V}$ one has $A^{W^{\lambda}}(\tilde{u})=A^{W}(u)$ and hence

$$
e^{-\frac{1}{2} s^{t} A^{W^{\lambda}}(\tilde{u}) s} \operatorname{det} A_{V V}^{W^{\lambda}}(\tilde{u})=e^{-\frac{1}{2} s^{t} A^{W}(u) s} \operatorname{det} A_{V V}^{W}(u) .
$$

Again for $i, j \in \tilde{V}$, we calculate

$$
\begin{aligned}
W_{i j}^{\lambda} \cosh \left(\tilde{u}_{i}-\tilde{u}_{j}\right) & =\frac{1}{2} W_{i j} \sqrt{1+\lambda_{i}} \sqrt{1+\lambda_{j}}\left(e^{\tilde{u}_{i}-\tilde{u}_{j}}+e^{\tilde{u}_{j}-\tilde{u}_{i}}\right) \\
& =\frac{1}{2} W_{i j} \sqrt{1+\lambda_{i}} \sqrt{1+\lambda_{j}}\left(\sqrt{\frac{1+\lambda_{j}}{1+\lambda_{i}}} e^{u_{i}-u_{j}}+\sqrt{\frac{1+\lambda_{i}}{1+\lambda_{j}}} e^{u_{j}-u_{i}}\right) \\
& =\frac{1}{2} W_{i j}\left(\left(1+\lambda_{j}\right) e^{u_{i}-u_{j}}+\left(1+\lambda_{i}\right) e^{u_{j}-u_{i}}\right) \\
& =W_{i j} \cosh \left(u_{i}-u_{j}\right)+\frac{1}{2} W_{i j}\left(\lambda_{j} e^{u_{i}-u_{j}}+\lambda_{i} e^{u_{j}-u_{i}}\right) .
\end{aligned}
$$

Summing this over all edges $i \sim j$ and using $W_{i j}=0$ for $i \not j$, we get

$$
\begin{aligned}
& \sum_{(i \sim j) \in \tilde{E}} W_{i j}^{\lambda} \cosh \left(\tilde{u}_{i}-\tilde{u}_{j}\right) \\
= & \sum_{(i \sim j) \in \tilde{E}} W_{i j} \cosh \left(u_{i}-u_{j}\right)+\frac{1}{2} \sum_{(i \sim j) \in \tilde{E}} W_{i j}\left(\lambda_{j} e^{u_{i}-u_{j}}+\lambda_{i} e^{u_{j}-u_{i}}\right) \\
= & \sum_{(i \sim j) \in \tilde{E}} W_{i j} \cosh \left(u_{i}-u_{j}\right)+\frac{1}{2} \sum_{j \in \tilde{V}} \lambda_{j} \sum_{i \in \tilde{V}} W_{i j} e^{u_{i}-u_{j}} \\
= & \sum_{(i \sim j) \in \tilde{E}} W_{i j} \cosh \left(u_{i}-u_{j}\right)+\sum_{j \in V} \lambda_{j} \beta_{j}^{W}(u),
\end{aligned}
$$

where in the last line we used $\lambda_{\delta}=0$. Therefore,

$$
\begin{aligned}
& \prod_{(i \sim j) \in \tilde{E}} e^{-W_{i j}^{\lambda}\left[\cosh \left(\tilde{u}_{i}-\tilde{u}_{j}\right)-1\right]} \\
= & \prod_{(i \sim j) \in \tilde{E}} e^{W_{i j}^{\lambda}-W_{i j}} \prod_{(i \sim j) \in \tilde{E}} e^{-W_{i j}\left[\cosh \left(u_{i}-u_{j}\right)-1\right]} \prod_{j \in V} e^{-\lambda_{j} \beta_{j}^{W}(u)} .
\end{aligned}
$$

Finally,

$$
\prod_{j \in V} e^{-\tilde{u}_{j}}=\prod_{j \in \tilde{V}} e^{-\tilde{u}_{j}}=\prod_{j \in \tilde{V}} \sqrt{1+\lambda_{j}} e^{-u_{j}},
$$

where we extended the product $\prod_{j \in V}$ to $\prod_{j \in \tilde{V}}$ using $\tilde{u}_{\delta}=0$. Substituting formulas (3.6), (3.9), and (3.10) into (3.5), claim (3.3) follows.

The following corollary gives a short alternative derivation of the Laplace transform of the random vector $\left(\beta_{i}\right)_{i \in V}$. It is a special case of Theorem 2.1 and also one of the ingredients for the proof of this theorem. 
Corollary 3.2 (Laplace transform of $\beta$, Sabot, Tarrès, and Zeng in Sabot et al., 2017+).

The function $\mathcal{L}^{W}$, defined in formula (2.9), is the Laplace transform of the random vector $\beta=\left(\beta_{i}\right)_{i \in V}$ :

$$
\mathbb{E}_{\mu^{W}}\left[e^{-\left\langle\lambda_{V}, \beta\right\rangle}\right]=\mathcal{L}^{W}(\lambda)=\prod_{(i \sim j) \in \tilde{E}} e^{W_{i j}\left(1-\sqrt{1+\lambda_{i}} \sqrt{1+\lambda_{j}}\right)} \prod_{i \in V} \frac{1}{\sqrt{1+\lambda_{i}}}
$$

for all $\lambda \in \Lambda_{V}$.

Proof: Integrating both sides of (3.3) over $\Omega_{V}$, the claim follows from the fact that the image measure $S_{\lambda} \mu^{W^{\lambda}}$ is a probability measure on $\Omega_{V}$.

The following corollary contains the previous one as special case $g=1$ :

Corollary 3.3. For any random variable $g: \Omega_{V} \rightarrow \mathbb{R}$ and any $\lambda \in \Lambda_{V}$, one has

$$
\mathbb{E}_{\mu^{W}}\left[g e^{-\left\langle\lambda_{V}, \beta\right\rangle}\right]=\mathcal{L}^{W}(\lambda) \mathbb{E}_{\mu^{W \lambda}}\left[g \circ S_{\lambda}\right]
$$

in the sense that the left-hand side exists if and only if the right-hand side exists.

Proof: Using Corollary 3.2, we rewrite claim (3.3) of Theorem 3.1 in the form

$$
e^{-\left\langle\lambda_{V}, \beta\right\rangle}=\mathcal{L}^{W}(\lambda) \frac{d\left(S_{\lambda} \mu^{W^{\lambda}}\right)}{d \mu^{W}} .
$$

This yields the claim as follows:

$$
\begin{aligned}
\mathbb{E}_{\mu W}\left[g e^{-\left\langle\lambda_{V}, \beta\right\rangle}\right] & =\mathcal{L}^{W}(\lambda) \mathbb{E}_{\mu^{W}}\left[g \frac{d\left(S_{\lambda} \mu^{W^{\lambda}}\right)}{d \mu^{W}}\right] \\
& =\mathcal{L}^{W}(\lambda) \mathbb{E}_{S_{\lambda} \mu^{W^{\lambda}}}[g]=\mathcal{L}^{W}(\lambda) \mathbb{E}_{\mu^{W^{\lambda}}}\left[g \circ S_{\lambda}\right] .
\end{aligned}
$$

Example 3.4. Taking $g(u, s)=e^{u_{k}}$ for any $k \in \tilde{V}$, this corollary gives

$$
\mathbb{E}_{\mu^{W}}\left[e^{u_{k}} e^{-\left\langle\lambda_{V}, \beta(u)\right\rangle}\right]=\mathcal{L}^{W}(\lambda) \sqrt{1+\lambda_{k}} .
$$

Indeed, using

$$
g\left(S_{\lambda}(u, s)\right)=e^{u_{k}+\log \sqrt{1+\lambda_{k}}}=\sqrt{1+\lambda_{k}} e^{u_{k}},
$$

formula (3.12) reduces to formula (3.15) as follows

$$
\mathbb{E}_{\mu^{W}}\left[e^{u_{k}} e^{-\left\langle\lambda_{V}, \beta(u)\right\rangle}\right]=\mathcal{L}^{W}(\lambda) \sqrt{1+\lambda_{k}} \mathbb{E}_{\mu^{W^{\lambda}}}\left[e^{u_{k}}\right]=\mathcal{L}^{W}(\lambda) \sqrt{1+\lambda_{k}} .
$$

The last equality follows from formula (B.3) in Appendix B of Disertori et al. (2010), which shows $\mathbb{E}_{\mu^{W^{\lambda}}}\left[e^{u_{k}}\right]=1$. It is also a consequence of Corollary 5.3 below; cf. formula (5.26). 


\subsection{Measurability.}

Proof of Lemma 2.3: Since $u_{\delta}=0$ we only need to consider $\left(u_{i}\right)_{i \in V}$. Given $u \in \mathbb{R}^{\tilde{V}}$ with $u_{\delta}=0$, the definition (2.6) of $\beta_{i}=\beta_{i}(u)$ can be reorganized as

$$
2 \beta_{i} e^{u_{i}}-\sum_{j \in V} W_{i j} e^{u_{j}}=W_{i \delta}
$$

Recall from (2.11), $\left(H_{\beta(u)}\right)_{i j}=2 \beta_{i}(u) \delta_{i j}-W_{i j}=e^{-u_{i}} A_{i j}^{W}(u) e^{-u_{j}}$ for all $i, j \in V$ and $H_{\beta}$ is invertible. Using the notations $e_{V}^{u}=\left(e^{u_{i}}\right)_{i \in V}$ and $W_{V \delta}=\left(W_{i \delta}\right)_{i \in V}$, equation (3.18) above becomes $H_{\beta} e_{V}^{u}=W_{V \delta}$ or equivalently for $i \in V$

$$
e^{u_{i}}=\left(H_{\beta}^{-1} W_{V \delta}\right)_{i}=e^{f_{V}^{W}\left(\beta^{V}\right)_{i}} .
$$

Thus, $e_{V}^{u}$ is $\sigma\left(\beta^{V}\right)$-measurable. The claim follows.

Remark. In our setup, starting with $H^{2 \mid 2}$, it is a priori clear that $e_{V}^{u}$ has positive entries. As a consequence, $\log \left(H_{\beta}^{-1} W_{V \delta}\right)$ is well-defined. In contrast to this, Sabot and Zeng (2015) starts with the distribution of the $\beta$ 's. There, additional arguments are needed to insure that this $\log$ is indeed well-defined.

\section{First martingale}

Proof of Lemma 2.4 - Kolmogorov consistency: Using Corollary 3.2, we can calculate both Laplace transforms:

$$
\begin{aligned}
\mathcal{L}_{n+1}^{W}(\lambda) & =\prod_{(i \sim j) \in \tilde{E}_{n+1}} e^{W_{i j}^{(n+1)}\left(1-\sqrt{1+\lambda_{i}} \sqrt{1+\lambda_{j}}\right)} \prod_{i \in V_{n+1}} \frac{1}{\sqrt{1+\lambda_{i}}}, \\
\mathcal{L}_{n}^{W}(\lambda) & =\prod_{(i \sim j) \in \tilde{E}_{n}} e^{W_{i j}^{(n)}\left(1-\sqrt{1+\lambda_{i}} \sqrt{1+\lambda_{j}}\right)} \prod_{i \in V_{n}} \frac{1}{\sqrt{1+\lambda_{i}}} .
\end{aligned}
$$

Since $\lambda_{i}=0$ for all $i \in V_{n+1} \backslash V_{n}$, the last product in (4.1) agrees with the last product in (4.2). It remains to consider the product over edges. Let $(i \sim j) \in \tilde{E}_{n+1}$. We distinguish several cases.

Case 1: $i \in V_{n}$ and $j \in V_{n}$. Then $(i \sim j) \in \tilde{E}_{n}$ and $W_{i j}^{(n+1)}=W_{i j}=W_{i j}^{(n)}$. Thus the contribution of this edge is the same in (4.1) and (4.2).

Case 2: $i \in \tilde{V}_{n+1} \backslash V_{n}$ and $j \in \tilde{V}_{n+1} \backslash V_{n}$. Then $W_{i j}^{(n+1)}\left(1-\sqrt{1+\lambda_{i}} \sqrt{1+\lambda_{j}}\right)=0$ because $\lambda_{i}=\lambda_{j}=0$. Furthermore, $(i \sim j) \notin \tilde{E}_{n}$. Thus, $i \sim j$ does not contribute. Case 3: $i \in V_{n}$ and $j \in \tilde{V}_{n+1} \backslash V_{n}$. For a fixed $i \in V_{n}$, we calculate

$$
\begin{aligned}
& \sum_{\substack{j \in \tilde{V}_{n+1} \backslash V_{n}: \\
(i \sim j) \in \tilde{E}_{n+1}}} W_{i j}^{(n+1)}\left(1-\sqrt{1+\lambda_{i}} \sqrt{1+\lambda_{j}}\right) \\
= & {\left[W_{i \delta_{n+1}}^{(n+1)}+\sum_{j \in V_{n+1} \backslash V_{n}} W_{i j}\right]\left(1-\sqrt{1+\lambda_{i}}\right) } \\
= & {\left[\sum_{j \in V_{\infty} \backslash V_{n}} W_{i j}\right]\left(1-\sqrt{1+\lambda_{i}}\right)=W_{i \delta_{n}}^{(n)}\left(1-\sqrt{1+\lambda_{i}}\right) . }
\end{aligned}
$$

This is the contribution of the edge $\left(i \sim \delta_{n}\right) \in \tilde{E}_{n}$ to (4.2).

Thus the products in (4.1) and (4.2) agree and the claim is proved. 
Proof of Theorem 2.5 - Martingale $e^{u}$ : Given $n \in \mathbb{N}$, it suffices to consider $k \in$ $V_{n+1}$, since otherwise $u_{k}^{(n+1)}=u_{k}^{(n)}=0$ and (2.24) is trivially satisfied. Note that $u_{k}^{(n)}=u_{\delta_{n}}^{(n)}=0$ for $k \in V_{n+1} \backslash V_{n}$. By its definition (2.22), $u_{k}^{(n)}$ is $\mathcal{F}_{n}$-measurable. It remains to prove

$$
\mathbb{E}_{\mu_{\infty}^{W}}\left[e^{u_{k}^{(n+1)}} g\left(\boldsymbol{\beta}^{(n)}\right)\right]=\mathbb{E}_{\mu_{\infty}^{W}}\left[e^{u_{k}^{(n)}} g\left(\boldsymbol{\beta}^{(n)}\right)\right]
$$

for any measurable function $g: \mathbb{R}^{V_{n}} \rightarrow[0, \infty)$. For any given $c \in \mathbb{R}$, the uniqueness theorem for Laplace transforms allows us to restrict the claim to test functions $g\left(\boldsymbol{\beta}^{(n)}\right)=\prod_{j \in V_{n}} e^{-\lambda_{j} \boldsymbol{\beta}_{j}}$ with $\lambda_{j}>c$ for all $j \in V_{n}$

$$
\mathbb{E}_{\mu \infty}\left[e^{u_{k}^{(n+1)}} \prod_{j \in V_{n}} e^{-\lambda_{j} \boldsymbol{\beta}_{j}}\right]=\mathbb{E}_{\mu \infty}\left[e^{u_{k}^{(n)}} \prod_{j \in V_{n}} e^{-\lambda_{j} \boldsymbol{\beta}_{j}}\right],
$$

as long as all these expectations are finite. As explained below formula (2.22), the law of $u^{(n)}$ with respect to $\mu_{\infty}^{W}$ coincides with the law of $u=\left(u_{i}\right)_{i \in \tilde{V}_{n}}$ with respect to $\mu_{n}^{W}$. In analogy to $(2.23)$, we define $u^{\prime}: \Omega_{V_{n}} \rightarrow \mathbb{R}^{V_{n+1}}$ by

$$
u_{k}^{\prime}= \begin{cases}u_{k}, & \text { if } k \in V_{n}, \\ u_{\delta_{n}}=0, & \text { if } k \in V_{n+1} \backslash V_{n} .\end{cases}
$$

Then, claim (4.5) is equivalent to

$$
\mathbb{E}_{\mu_{n+1}^{W}}\left[e^{u_{k}} \prod_{j \in V_{n}} e^{-\lambda_{j} \beta_{j}^{V_{n}+1}(u)}\right]=\mathbb{E}_{\mu_{n}^{W}}\left[e^{u_{k}^{\prime}} \prod_{j \in V_{n}} e^{-\lambda_{j} \beta_{j}^{V_{n}}(u)}\right] .
$$

For $c=-1$, Corollary 3.3 and Example 3.4 imply that these expectations are finite; hence the same is true for the expectations in (4.5).

Set $\lambda_{i}=0$ for all $i \in \tilde{V}_{n+1} \backslash V_{n}$. Using Example 3.4 and Lemma 2.4, we obtain the claim (4.7) in both cases, $k \in V_{n}$ or $k \in V_{n+1} \backslash V_{n}$, as follows:

$$
\begin{aligned}
& \mathbb{E}_{\mu_{n+1}^{W}}\left[e^{u_{k}} \prod_{j \in V_{n}} e^{-\lambda_{j} \beta_{j}^{V_{n}+1}(u)}\right]=\mathbb{E}_{\mu_{n+1}^{W}}\left[e^{u_{k}} \prod_{j \in V_{n+1}} e^{-\lambda_{j} \beta_{j}^{V_{n+1}}(u)}\right] \\
& =\mathcal{L}_{n+1}^{W}(\lambda) \sqrt{1+\lambda_{k}}=\mathcal{L}_{n}^{W}(\lambda) \sqrt{1+\lambda_{k}}=\mathbb{E}_{\mu_{n}^{W}}\left[e^{u_{k}^{\prime}} \prod_{j \in V_{n}} e^{-\lambda_{j} \beta_{j}^{V_{n}}(u)}\right] .
\end{aligned}
$$

\section{Using (super-)symmetries of the model}

Let $\tilde{\mathcal{G}}=(\tilde{V}, \tilde{E})$ be a finite graph as described at the beginning of Section 2 . Disertori, Spencer, and Zirnbauer in Disertori et al. (2010) use an alternative representation in terms of Grassmann variables of the $H^{2 \mid 2}$ measure $\mu^{W}$ defined in (2.4). It has the advantage of making the internal symmetries and supersymmetries of the model visible. Since we are using these symmetries in the remainder, we briefly review this alternative representation; cf. Section 2.2 of Disertori et al. (2010). Let $\bar{\psi}_{i}, \psi_{i}, i \in V$, be independent Grassmann variables, and let $\bar{\psi}_{\delta}=0=\psi_{\delta}$. Motivated by $\bar{\psi}_{i}^{2}=\psi_{i}^{2}=0$, a smooth superfunction $f(u, s, \bar{\psi}, \psi)$ is defined to be a polynomial 
in the Grassmann variables

$$
f(u, s, \bar{\psi}, \psi)=\sum_{I, J \subseteq V} f_{I J}(u, s) \bar{\psi}_{I} \psi_{J},
$$

where the coefficients $f_{I J}$ are smooth functions of $u$ and $s$ and we abbreviate

$$
\bar{\psi}_{I}=\prod_{i \in I} \bar{\psi}_{i}, \quad \psi_{J}=\prod_{j \in J} \psi_{j}
$$

the products are understood with respect to some fixed arbitrary ordering of the vertex set $V$. Since each $\bar{\psi}_{i}$ or $\psi_{i}$ appears with degree at most one, $\prod_{i \in V} \partial_{\bar{\psi}_{i}} \partial_{\psi_{i}} f$ is a smooth function of $u$ and $s$ only.

The measure $\mu^{W}$ can be represented as follows

$$
\mu^{W}(d u d s)=\prod_{i \in V} e^{-u_{i}} \frac{d u_{i} d s_{i}}{2 \pi} \partial_{\bar{\psi}_{i}} \partial_{\psi_{i}} e^{\mathcal{S}}
$$

with the action

$$
\begin{aligned}
\mathcal{S} & =\mathcal{S}(u, s, \bar{\psi}, \psi) \\
= & -\sum_{(i \sim j) \in \tilde{E}} W_{i j}\left[\cosh \left(u_{i}-u_{j}\right)-1+\left[\frac{1}{2}\left(s_{i}-s_{j}\right)^{2}+\left(\bar{\psi}_{i}-\bar{\psi}_{j}\right)\left(\psi_{i}-\psi_{j}\right)\right] e^{u_{i}+u_{j}}\right] .
\end{aligned}
$$

Note that $e^{\mathcal{S}}=e^{\mathcal{S}(u, s, \bar{\psi}, \psi)}$ is a smooth function. Therefore, using the above argument, $\prod_{i \in V} \partial_{\bar{\psi}_{i}} \partial_{\psi_{i}} e^{\mathcal{S}}$ is a function of $u$ and $s$ only. Thus, $\mu^{W}$ is the marginal of the superintegration form

$$
\mathbb{E}_{D \mu^{W}}[f]=\int D \mu^{W} f:=\int_{\Omega_{V}} \prod_{i \in V} e^{-u_{i}} \frac{d u_{i} d s_{i}}{2 \pi} \partial_{\bar{\psi}_{i}} \partial_{\psi_{i}}\left(e^{\mathcal{S}} f(u, s, \bar{\psi}, \psi)\right)
$$

obtained by integrating the Grassmann variables out. This superexpectation is defined whenever $\prod_{i \in V} e^{-u_{i}} \partial_{\bar{\psi}_{i}} \partial_{\psi_{i}}\left(e^{\mathcal{S}} f(u, s, \bar{\psi}, \psi)\right)$ is an integrable function.

The internal (super-)symmetries of $D \mu^{W}$ are most easily seen in cartesian coordinates $x=\left(x_{i}\right)_{i \in \tilde{V}}, y=\left(y_{i}\right)_{i \in \tilde{V}}, z=\left(z_{i}\right)_{i \in \tilde{V}}, \xi=\left(\xi_{i}\right)_{i \in \tilde{V}}$, and $\eta=\left(\eta_{i}\right)_{i \in \tilde{V}}$ defined by

$$
\begin{aligned}
& x_{i}=\sinh u_{i}-\left(\frac{1}{2} s_{i}^{2}+\bar{\psi}_{i} \psi_{i}\right) e^{u_{i}}, \quad y_{i}=s_{i} e^{u_{i}}, \quad \xi_{i}=e^{u_{i}} \bar{\psi}_{i}, \quad \eta_{i}=e^{u_{i}} \psi_{i}, \\
& z_{i}=\sqrt{1+x_{i}^{2}+y_{i}^{2}+2 \xi_{i} \eta_{i}}=\cosh u_{i}+\left(\frac{1}{2} s_{i}^{2}+\bar{\psi}_{i} \psi_{i}\right) e^{u_{i}} .
\end{aligned}
$$

In particular, $x_{\delta}=y_{\delta}=\xi_{\delta}=\eta_{\delta}=0$ and $z_{\delta}=1$. A geometrical intuition of these coordinates is given in the appendix. As described in sections 2.1 and 2.2 of Disertori et al. (2010), the image $D \mu_{\text {cart }}^{W}$ of $D \mu^{W}$ under this supertransformation is given by formulas (2.5) and (2.6) of that paper:

$$
D \mu_{\text {cart }}^{W}=\left(\prod_{i \in V} \frac{d x_{i} d y_{i}}{2 \pi} \partial_{\xi_{i}} \partial_{\eta_{i}} \circ \frac{1}{z_{i}}\right) e^{\mathcal{S}_{\text {cart }}}
$$

with the transformed action $\mathcal{S}_{\text {cart }}=\mathcal{S}_{\text {cart }}(x, y, \xi, \eta)$ given by

$$
\mathcal{S}_{\text {cart }}=-\sum_{(i \sim j) \in \tilde{E}} W_{i j}\left(-1-x_{i} x_{j}-y_{i} y_{j}+z_{i} z_{j}-\xi_{i} \eta_{j}+\eta_{i} \xi_{j}\right) .
$$


In (5.8), the symbol $\circ$ means that the partial derivatives $\partial_{\xi_{i}} \partial_{\eta_{i}}$ act not only on $\frac{1}{z_{i}}$ and $e^{\mathcal{S}_{\text {cart }}}$, but also on the test function as follows:

$$
\begin{aligned}
& \mathbb{E}_{D \mu_{\text {cart }}^{W}}[f]=\int D \mu_{\text {cart }}^{W} f \\
:= & \int \prod_{i \in V} \frac{d x_{i} d y_{i}}{2 \pi} \partial_{\xi_{i}} \partial_{\eta_{i}}\left(\prod_{i \in V} \frac{1}{z_{i}} \cdot e^{\mathcal{S}_{\text {cart }}(x, y, \xi, \eta)} f(x, y, \xi, \eta)\right)
\end{aligned}
$$

To make the superintegration transformation from $D \mu_{\text {cart }}^{W}$ to $D \mu^{W}$ precise, let $\mathcal{A}(\xi, \eta)$ be the Grassmann algebra generated by the family of Grassmann variables $\xi_{i}, \eta_{i}, i \in V$. Let $\mathbb{S}_{\text {susy }}\left(\Omega_{V}, \xi, \eta\right)$ denote the space of superfunctions of the form

$$
\begin{aligned}
f_{\text {cart }}: & \Omega_{V} \rightarrow \mathcal{A}(\xi, \eta) \\
& (x, y) \mapsto f_{\text {cart }}(x, y, \xi, \eta)=\sum_{I, J \subseteq V} f_{I J}(x, y) \xi_{I} \eta_{J},
\end{aligned}
$$

where the coefficients $f_{I J}$ are Schwartz functions and the products $\xi_{I}$ and $\eta_{J}$ of Grassmann variables are defined in analogy to (5.2) as follows

$$
\xi_{I}=\prod_{i \in I} \xi_{i}, \quad \eta_{J}=\prod_{j \in J} \eta_{j} .
$$

After doing the change of coordinates given in (5.6), we obtain the test function in horospherical coordinates $f_{\text {hor }}: \Omega_{V} \rightarrow \mathcal{A}(\bar{\psi}, \psi)$,

$$
\begin{aligned}
(u, s) & \mapsto f_{\text {hor }}(u, s, \bar{\psi}, \psi) \\
& =f_{\text {cart }}(x(u, s, \bar{\psi}, \psi), y(u, s, \bar{\psi}, \psi), \xi(u, s, \bar{\psi}, \psi), \eta(u, s, \bar{\psi}, \psi)) .
\end{aligned}
$$

Then we have the following superintegration transformation formula. It is proved in the appendix.

Lemma 5.1. For any superfunction $f_{\text {cart }}(x, y, \xi, \eta)$ with the property $e^{\mathcal{S}_{\text {cart }}} f_{\text {cart }} \in$ $\mathbb{S}_{\text {susy }}\left(\Omega_{V}, \xi, \eta\right)$, we have

$$
\mathbb{E}_{D \mu_{\text {cart }}^{W}}\left[f_{\text {cart }}\right]=\mathbb{E}_{D \mu^{W}}\left[f_{\text {hor }}\right]
$$

In the following we will use only test superfunctions $f=f_{\text {cart }}$ which satisfy the assumption of this lemma.

Rotational symmetry. It is obvious that $D \mu_{\text {cart }}^{W}$ is invariant with respect to rotations in the $x y$-plane, $(x, y, \xi, \eta) \mapsto\left(x^{\alpha}, y^{\alpha}, \xi, \eta\right)$ with

$$
x^{\alpha}=x \cos \alpha-y \sin \alpha, \quad y^{\alpha}=x \sin \alpha+y \cos \alpha, \quad \text { for } \alpha \in \mathbb{R} .
$$

In horospherical coordinates $u, s, \bar{\psi}, \psi$ this symmetry is not so easy to describe and somehow hidden.

$Q$-supersymmetry. In Disertori et al. (2010), the invariance of the $H^{2 \mid 2}$-model with respect to the supersymmetry operator

$$
Q=\sum_{i \in V}\left(x_{i} \partial_{\eta_{i}}-y_{i} \partial_{\xi_{i}}+\xi_{i} \partial_{x_{i}}+\eta_{i} \partial_{y_{i}}\right)
$$

played a key role. From Proposition 2 in Appendix C of Disertori et al. (2010) we know that for any smooth superfunction $f=f(x, y, \xi, \eta)$ with $Q f=0$ and $e^{\mathcal{S}_{\text {cart }} f \in \mathbb{S}_{\text {susy }}\left(\Omega_{V}, \xi, \eta\right) \text {, one has }}$

$$
\mathbb{E}_{D \mu_{\text {cart }}^{W}}[f]=e^{\mathcal{S}_{\text {cart }}(o)} f(o)=f(o),
$$


where $o$ denotes the zero-field configuration $x=y=0, \xi=\eta=0$. In particular, the assumption $Q f=0$ is satisfied for smooth superfunctions of the form $f=f(z)$ because of $Q z_{i}=0$ for all $i \in V$.

These (super-)symmetries play the key role in the proof of the following lemma.

Lemma 5.2 (Ward identities). Let $f: \mathbb{R}^{2} \rightarrow \mathbb{C}$ be a harmonic function and $\theta \in \mathbb{R}^{\tilde{V}}$.

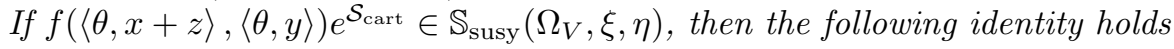

$$
\mathbb{E}_{D \mu_{\text {cart }}^{W}}[f(\langle\theta, x+z\rangle,\langle\theta, y\rangle)]=f(\langle\theta, 1\rangle, 0),
$$

where $\langle\theta, 1\rangle$ stands for $\sum_{i \in \tilde{V}} \theta_{i}$.

Note that the extension of $f$ to a superfunction is used in the expectation because $z$ defined in (5.7) involves Grassmann variables. This extension is denoted by the same symbol $f$.

Proof: By rotational symmetry of the model $H^{2 \mid 2}$ in the $x y$-plane, using the notation (5.15), we have

$$
\mathbb{E}_{D \mu_{\text {cart }}^{W}}[f(\langle\theta, x+z\rangle,\langle\theta, y\rangle)]=\mathbb{E}_{D \mu_{\text {cart }}^{W}}\left[f\left(\left\langle\theta, x^{\alpha}+z\right\rangle,\left\langle\theta, y^{\alpha}\right\rangle\right)\right]
$$

for any $\alpha \in \mathbb{R}$. Taking the average over $\alpha \in[0,2 \pi]$ and using the mean value theorem for the harmonic function $f$ yields

$$
\begin{aligned}
& \mathbb{E}_{D \mu_{\text {cart }}^{W}}[f(\langle\theta, x+z\rangle,\langle\theta, y\rangle)]=\frac{1}{2 \pi} \int_{0}^{2 \pi} \mathbb{E}_{D \mu_{\text {cart }}^{W}}\left[f\left(\left\langle\theta, x^{\alpha}+z\right\rangle,\left\langle\theta, y^{\alpha}\right\rangle\right)\right] d \alpha \\
& =\mathbb{E}_{D \mu_{\text {cart }}^{W}}\left[\frac{1}{2 \pi} \int_{0}^{2 \pi} f\left(\left\langle\theta, x^{\alpha}+z\right\rangle,\left\langle\theta, y^{\alpha}\right\rangle\right) d \alpha\right]=\mathbb{E}_{D \mu_{\text {cart }}^{W}}[f(\langle\theta, z\rangle, 0)] .
\end{aligned}
$$

Since $f(\langle\theta, z\rangle, 0)$ is a smooth superfunction of $z$, we have the supersymmetry

$$
Q f(\langle\theta, z\rangle, 0)=0 .
$$

The assumption $f(\langle\theta, x+z\rangle,\langle\theta, y\rangle) e^{\mathcal{S}_{\text {cart }}} \in \mathbb{S}_{\text {susy }}\left(\Omega_{V}, \xi, \eta\right)$ implies that we can apply Proposition 2 from Appendix $\mathrm{C}$ of Disertori et al. (2010), cited in (5.17), to the averaged superfunction $f(\langle\theta, z\rangle, 0)$. It yields

$$
\mathbb{E}_{D \mu_{\text {cart }}^{W}}[f(\langle\theta, z\rangle, 0)]=f(\langle\theta, 1\rangle, 0) .
$$

Corollary 5.3 (Ward identity for exp). For all $\theta \in(-\infty, 0]^{\tilde{V}}$, one has

$$
\mathbb{E}_{\mu w}\left[e^{\left\langle\theta, e^{u}(1+i s)\right\rangle}\right]=e^{\langle\theta, 1\rangle},
$$

using the abbreviation $e^{u}(1+i s)=\left(e^{u_{j}}\left(1+i s_{j}\right)\right)_{j \in \tilde{V}}$.

Proof: By Lemma B.1 in the appendix, $e^{\mathcal{S}_{\text {cart }}} \in \mathbb{S}_{\text {susy }}\left(\Omega_{V}, \xi, \eta\right)$. Note that all coefficients in the expansion (5.11) of $e^{\langle\theta, x+z+i y\rangle}$, together with all their derivatives of any order in $x$ and $y$, are of the form $p \cdot e^{\left\langle\theta, x+z^{\prime}+i y\right\rangle}$ with $z^{\prime}=\left(z_{i}^{\prime}=\right.$ $\left.\sqrt{1+x_{i}^{2}+y_{i}^{2}}\right)_{i \in \tilde{V}}$, where $p: \Omega_{V} \rightarrow \mathbb{R}$ are algebraic functions of $x$ and $y$, nowhere singular. Moreover, for any $\theta \in(-\infty, 0]^{\tilde{V}}$, the term $e^{\left\langle\theta, x+z^{\prime}+i y\right\rangle}$ is bounded. Therefore, $e^{\mathcal{S}_{\text {cart }}} e^{\langle\theta, x+z+i y\rangle} \in \mathbb{S}_{\text {susy }}\left(\Omega_{V}, \xi, \eta\right)$, as well. Thus, we can apply Lemma 5.1 as follows:

$$
\mathbb{E}_{\mu^{W}}\left[e^{\left\langle\theta, e^{u}(1+i s)\right\rangle}\right]=\mathbb{E}_{D \mu_{\text {cart }}^{W}}\left[e^{\langle\theta, x+z+i y\rangle}\right],
$$


since from (5.6) and (5.7), we know $x_{j}+z_{j}=e^{u_{j}}$ and $y_{j}=s_{j} e^{u_{j}}$. We apply now Lemma 5.2 to the holomorphic (and hence harmonic) function $f=\exp : \mathbb{R}^{2}=\mathbb{C} \rightarrow$ $\mathbb{C}, f(x, y)=e^{x+i y}$. We obtain

$$
\mathbb{E}_{D \mu_{\text {cart }}^{W}}\left[e^{\langle\theta, x+z+i y\rangle}\right]=e^{\langle\theta, 1\rangle},
$$

which proves the claim (5.23).

Remark. As a consequence of Corollary 5.3, we obtain for all vertices $k, l, m \in \tilde{V}$,

$$
\begin{aligned}
& \mathbb{E}_{\mu^{W}}\left[e^{u_{k}}\right]=1, \\
& \mathbb{E}_{\mu^{W}}\left[e^{u_{k}+u_{l}}\left(1-s_{k} s_{l}\right)\right]=1, \\
& \mathbb{E}_{\mu^{W}}\left[e^{u_{k}+u_{l}+u_{m}}\left(1-s_{k} s_{l}-s_{k} s_{m}-s_{l} s_{m}\right)\right]=1 .
\end{aligned}
$$

More generally, for any $m \in \mathbb{N}$ and any $i_{1}, \ldots, i_{m} \in \tilde{V}$,

$$
\mathbb{E}_{\mu^{W}}\left[e^{\sum_{j=1}^{m} u_{i_{j}}} \sum_{\substack{I \subseteq\{1, \ldots, m\}: \\|I| \text { even }}}(-1)^{|I| / 2} \prod_{k \in I} s_{i_{k}}\right]=1 .
$$

Indeed, given $m \in \mathbb{N}$ and $i_{1}, \ldots, i_{m} \in \tilde{V}_{n}$, we take the left derivative $\partial_{\theta_{i_{1}}} \ldots \partial_{\theta_{i_{m}}}$ at $\theta=0$ of (5.23) to get

$$
\mathbb{E}_{\mu^{W}}\left[\prod_{k=1}^{m} e^{u_{i_{k}}\left(1+i s_{i_{k}}\right)}\right]=1 .
$$

Note that the hypothesis $\theta_{i} \leq 0$ allows us to interchange expectations and partial derivatives. Expanding the product and taking the real part of this equation gives formula (5.29). The cases $m=1,2,3$ of this formula may be written in the form (5.26), (5.27), and (5.28), respectively.

Using $u_{\delta}=s_{\delta}=0$, note that the $(m+1)$-st instance of formula (5.29) contains the $m$-th instance as special case $i_{m+1}=\delta$.

\section{A hierarchy of martingales}

For a finite graph $\tilde{\mathcal{G}}=(\tilde{V}, \tilde{E})$ with $\delta \in \tilde{V}$, recall the definitions (2.3) of the matrix $A^{W}$ and (2.5) of the Green's function $\hat{G}$. We remind that the Gaussian part in the measure $\mu^{W}$ defined in (2.4) can be rewritten as

$$
\prod_{(i \sim j) \in \tilde{E}} e^{-\frac{1}{2} W_{i j}\left(s_{i}-s_{j}\right)^{2} e^{u_{i}+u_{j}}}=e^{-\frac{1}{2} s^{t} A^{W}(u) s} .
$$

Therefore, we have the following representations of the Green's function as conditional expectation:

$$
\begin{aligned}
\hat{G}_{i j} & =\mathbb{E}_{\mu^{W}}\left[s_{i} s_{j} e^{u_{i}+u_{j}} \mid u\right] \quad \mu^{W} \text {-a.s., } \quad \text { for all } i, j \in \tilde{V}, \\
e^{-\frac{1}{2}\langle\theta, \hat{G} \theta\rangle} & =\mathbb{E}_{\mu^{W}}\left[e^{i\left\langle\theta, s e^{u}\right\rangle} \mid u\right] \quad \mu^{W} \text {-a.s., } \quad \text { for any } \theta \in \mathbb{R}^{\tilde{V}} .
\end{aligned}
$$

Note that $s_{i} s_{j} e^{u_{i}+u_{j}} \in L^{p}\left(\Omega_{V}, \mu^{W}\right)$ implies $\hat{G}_{i j} \in L^{p}\left(\Omega_{V}, \mu^{W}\right)$ for all $p \in[1, \infty)$.

To prove Theorem 2.6, we need some preliminaries. Since the martingale $M_{n}(\theta)$ in that theorem involves the Green's function and we use the preceding representation as a conditional expectation, we need the following variant of Corollary 3.3. 
Lemma 6.1. For any random variable $g: \Omega_{V} \rightarrow \mathbb{R}$ and any $\lambda \in \Lambda_{V}$, one has

$$
\mathbb{E}_{\mu^{W}}\left[\mathbb{E}_{\mu^{W}}[g \mid u] e^{-\left\langle\lambda_{V}, \beta\right\rangle}\right]=\mathcal{L}^{W}(\lambda) \mathbb{E}_{\mu^{W^{\lambda}}}\left[g \circ S_{\lambda}\right]
$$

in the sense that the left-hand side exists if and only if the right-hand side exists.

Proof: This follows immediately from Corollary 3.3 because $\beta$ is a function of $u$, but not of $s$.

From this lemma we get immediately the proof of Theorem 2.1.

Proof of Theorem 2.1: Using the conditional Laplace transform (6.3) and the fact that $\beta$ is a function of $u$ only, we can rewrite the claim with the function $g_{\theta}(u, s)=$ $e^{\left\langle\theta, e^{u}(1+i s)\right\rangle}$ as follows:

$$
\mathbb{E}_{\mu^{W}}\left[\mathbb{E}_{\mu^{W}}\left[g_{\theta} \mid u\right] e^{-\left\langle\lambda_{V}, \beta\right\rangle}\right]=\mathcal{L}^{W}(\lambda) e^{\langle\theta, \sqrt{1+\lambda}\rangle} .
$$

We apply Lemma 6.1 to the left-hand side. Observe that

$$
g_{\theta}\left(S_{\lambda}(u, s)\right)=e^{\left\langle\theta, \sqrt{1+\lambda} e^{u}(1+i s)\right\rangle}=g_{\theta \sqrt{1+\lambda}}(u, s) .
$$

Since $\mathbb{E}_{\mu^{W^{\lambda}}}\left[g_{\theta \sqrt{1+\lambda}}\right]=e^{\langle\theta, \sqrt{1+\lambda}\rangle}$ by Corollary 5.3 , the claim follows.

With these tools we can now prove the main result of this section.

Proof of Theorem 2.6 - Generating martingale: The proof follows the same lines as the proof of Theorem 2.5. Recall that $\hat{G}^{(n)}$ is a function of $u^{(n)}$. Consequently, by Lemma 2.3, $\hat{G}^{(n)}$ and hence $M^{(n)}(\theta)$ are $\mathcal{F}_{n}$-measurable. To prove the martingale property, it suffices to show

$$
\mathbb{E}_{\mu_{\infty}^{W}}\left[M^{(n+1)}(\theta) \prod_{j \in V_{n}} e^{-\lambda_{j} \boldsymbol{\beta}_{j}}\right]=\mathbb{E}_{\mu_{\infty}^{W}}\left[M^{(n)}(\theta) \prod_{j \in V_{n}} e^{-\lambda_{j} \boldsymbol{\beta}_{j}}\right]
$$

for all $\lambda_{i}>-1, i \in V_{n}$. Recall that by the construction in Section 2.2 the law of $\beta^{(n)}$ with respect to $\mu_{\infty}^{W}$ coincides with the law of $\beta^{V_{n}}$ with respect to $\mu_{n}^{W}$. Hence, we rewrite the claim (6.7) in the form

$$
\mathbb{E}_{\mu_{n+1}^{W}}\left[\tilde{M}^{(n+1)}(\theta) \prod_{j \in V_{n}} e^{-\lambda_{j} \beta_{j}^{V_{n+1}}(u)}\right]=\mathbb{E}_{\mu_{n}^{W}}\left[\tilde{M}^{(n)}(\theta) \prod_{j \in V_{n}} e^{-\lambda_{j} \beta_{j}^{V_{n}}(u)}\right],
$$

with the following variant of $M^{(n)}(\theta)$

$$
\tilde{M}^{(n)}(\theta): \Omega_{V_{n}} \rightarrow \mathbb{R}, \quad \tilde{M}^{(n)}(\theta)=e^{\left\langle\theta^{(n)}, e^{u}\right\rangle-\frac{1}{2}\left\langle\theta^{(n)}, \hat{G}^{V_{n}}(u) \theta^{(n)}\right\rangle} .
$$

Compare (6.8) with the similar claim (4.7). Set $\lambda_{i}=0$ for $i \in \tilde{V}_{n+1} \backslash V_{n}$. Using Theorem 2.1, claim (6.8) is equivalent to

$$
\mathcal{L}_{n+1}^{W}(\lambda) e^{\left\langle\theta^{(n+1)}, \sqrt{1+\lambda}\right\rangle}=\mathcal{L}_{n}^{W}(\lambda) e^{\left\langle\theta^{(n)}, \sqrt{1+\lambda}\right\rangle} .
$$


By Lemma 2.4, one has $\mathcal{L}_{n+1}^{W}(\lambda)=\mathcal{L}_{n}^{W}(\lambda)$. We calculate the remaining factors using the definition (2.25) of $\theta^{(i)}, i \in\{n, n+1\}$ :

$$
\begin{aligned}
\left\langle\theta^{(n)}, \sqrt{1+\lambda}\right\rangle & =\sum_{i \in V_{n}} \theta_{i} \sqrt{1+\lambda_{i}}+\theta_{\delta_{n}}^{(n)}=\sum_{i \in V_{n}} \theta_{i} \sqrt{1+\lambda_{i}}+\sum_{j \in V_{\infty} \backslash V_{n}} \theta_{j}, \\
\left\langle\theta^{(n+1)}, \sqrt{1+\lambda}\right\rangle & =\sum_{i \in V_{n+1}} \theta_{i} \sqrt{1+\lambda_{i}}+\sum_{j \in V_{\infty} \backslash V_{n+1}} \theta_{j}=\left\langle\theta^{(n)}, \sqrt{1+\lambda}\right\rangle,
\end{aligned}
$$

where in the last step we use $\lambda_{i}=0$ for $i \in \tilde{V}_{n+1} \backslash V_{n}$. Thus, equality (6.10) holds and the martingale property is shown.

Proof of Corollary 2.7 - Hierarchy of martingales: The random variable $M_{i_{1}, \ldots, i_{m}}^{(n)}$ is $\mathcal{F}_{n}$-measurable as a function of $u^{(n)}$ and $\hat{G}^{(n)}$. The martingale property for $M_{i_{1}, \ldots, i_{m}}^{(n)}$ is obtained by expanding the corresponding property for $M^{(n)}(\theta)$ from Theorem 2.6 around $\theta=0$, as follows. We rewrite the martingale property for $M^{(n)}(\theta)$ in the following form:

$$
\mathbb{E}_{\mu_{\infty}^{W}}\left[M^{(n+1)}(\theta) 1_{A}\left(\boldsymbol{\beta}^{(n)}\right)\right]=\mathbb{E}_{\mu_{\infty}^{W}}\left[M^{(n)}(\theta) 1_{A}\left(\boldsymbol{\beta}^{(n)}\right)\right]
$$

for any $A \in \mathcal{B}\left(\mathbb{R}^{V_{n}}\right), n \in \mathbb{N}, \theta \in(-\infty, 0]^{\left(V_{\infty}\right)}$, using the notation $\boldsymbol{\beta}^{(n)}=\left(\boldsymbol{\beta}_{i}\right)_{i \in V_{n}}$ again. We take $m$ (left) partial derivatives of this equation with respect to $\theta$; note that the hypothesis $\theta_{i} \leq 0$ and the fact that all moments of $\hat{G}^{(n)}$ are finite allow us to interchange expectations and partial derivatives. This yields

$$
\mathbb{E}_{\mu_{\infty}^{W}}\left[\partial_{\theta_{i_{1}}} \ldots \partial_{\theta_{i_{m}}} M^{(n+1)}(\theta) 1_{A}\left(\boldsymbol{\beta}^{(n)}\right)\right]=\mathbb{E}_{\mu_{\infty}^{W}}\left[\partial_{\theta_{i_{1}}} \ldots \partial_{\theta_{i_{m}}} M^{(n)}(\theta) 1_{A}\left(\boldsymbol{\beta}^{(n)}\right)\right]
$$

We use the well-known Isserlis-Wick-formula for $I \subseteq\{1, \ldots, m\}$ in the form

$$
\left.\left(\prod_{i \in I} \partial_{\theta_{j_{i}}}\right) e^{-\frac{1}{2}\left\langle\theta^{(n)}, \hat{G}^{(n)} \theta^{(n)}\right\rangle}\right|_{\theta=0}=\sum_{\mathcal{I} \in \mathcal{P}_{2}(I)} \prod_{\{k, l\} \in \mathcal{I}}\left(-\hat{G}_{k, l}^{(n)}\right) .
$$

The sum on the right-hand side is empty for sets $I$ with odd cardinality. Taking the iterated derivative of $M_{n}(\theta)$ as defined in (2.26), using the Leibniz rule and (6.15), we get

$$
\begin{aligned}
& \left.\partial_{\theta_{i_{1}}} \ldots \partial_{\theta_{i_{m}}} M^{(n)}(\theta)\right|_{\theta=0} \\
& =\left.\sum_{\substack{I \subseteq\{1, \ldots, m\} \\
|I| \text { even }}} e^{\sum_{k \in\{1, \ldots, m\} \backslash I} u_{i_{k}}^{(n)}}\left(\prod_{i \in I} \partial_{\theta_{j_{i}}}\right) e^{-\frac{1}{2}\left\langle\theta^{(n)}, \hat{G}^{(n)} \theta^{(n)}\right\rangle}\right|_{\theta=0}=M_{i_{1}, \ldots, i_{m}}^{(n)}
\end{aligned}
$$

Inserting this and the corresponding identity for $M^{(n+1)}(\theta)$ into (6.14) yields the martingale property for $M_{i_{1}, \ldots, i_{m}}^{(n)}, n \in \mathbb{N}$, in the form

$$
\mathbb{E}_{\mu_{\infty}^{W}}\left[M_{i_{1}, \ldots, i_{m}}^{(n+1)} 1_{A}\left(\boldsymbol{\beta}^{(n)}\right)\right]=\mathbb{E}_{\mu W}\left[M_{i_{1}, \ldots, i_{m}}^{(n)} 1_{A}\left(\boldsymbol{\beta}^{(n)}\right)\right] .
$$




\section{Proof of Letac's formula}

7.1. A special case. We consider first the simpler case $\phi=1$, i.e. $\phi_{i}=1$ for all $i \in V$. We will see later that the general case follows by a scaling argument.

It is shown in Theorem 1 in Sabot et al. $(2017+)$ that the following is a probability measure on $\mathbb{R}^{V}$ :

$$
\nu(d \beta)=\nu^{W, 1}(d \beta)=1_{\left\{H_{\beta}>0\right\}}\left(\frac{2}{\pi}\right)^{\frac{|V|}{2}} e^{-\langle 1, \beta\rangle} \prod_{(i \sim j) \in E} e^{W_{i j}} \frac{1}{\sqrt{\operatorname{det} H_{\beta}}} d \beta .
$$

Using the measure $\nu$, we obtain the relation

$$
\begin{aligned}
L & :=\left(\frac{2}{\pi}\right)^{\frac{|V|}{2}} \int_{\left\{b \in \mathbb{R}^{V}: H_{b}>0\right\}} \frac{e^{-\frac{1}{2}\left(\left\langle 1, H_{b} 1\right\rangle+\left\langle\theta, H_{b}^{-1} \theta\right\rangle\right)}}{\sqrt{\operatorname{det} H_{b}}} d b \\
& =\mathbb{E}_{\nu}\left[e^{\langle 1, \beta\rangle-\frac{1}{2}\left(\left\langle 1, H_{\beta} 1\right\rangle+\left\langle\theta, H_{\beta}^{-1} \theta\right\rangle\right)}\right] \prod_{(i \sim j) \in E} e^{-W_{i j}}=\mathbb{E}_{\nu}\left[e^{-\frac{1}{2}\left\langle\theta, H_{\beta}^{-1} \theta\right\rangle}\right],
\end{aligned}
$$

where, in the last equality, we have used

$$
\langle 1, \beta\rangle-\frac{1}{2}\left\langle 1, H_{\beta} 1\right\rangle-\sum_{(i \sim j) \in E} W_{i j}=\frac{1}{2} \sum_{i, j \in V} W_{i j}-\sum_{(i \sim j) \in E} W_{i j}=0 .
$$

The problem then reduces to evaluate $\mathbb{E}_{\nu}\left[e^{-\frac{1}{2}\left\langle\theta, H_{\beta}^{-1} \theta\right\rangle}\right]$. This is done in three steps.

Step 1. Let $\operatorname{law}_{\nu}(\beta)$ denote the law of $\beta=\left(\beta_{i}\right)_{i \in V}$ with respect to $\nu$. In Corollary 2 of Sabot et al. (2017+), Sabot, Tarrès, and Zeng express law ${ }_{\nu}(\beta)$ using $\beta$ defined in analogy to (2.6) and an additional independent gamma distributed random variable, associated to a special vertex inside $V$.

In contrast to this, here we consider again the enlarged vertex set $\tilde{V}=V \cup\{\delta\}$ and the $H^{2 \mid 2}$ measure $\mu^{W}$, defined in (2.4), on the enlarged graph $(\tilde{V}, \tilde{E})$. We may assume the vertex $\delta \in \tilde{V} \backslash V$ is connected to a single vertex $\ell \in V$,

$$
\tilde{E}=E \cup\{\ell \sim \delta\}, \quad E=\tilde{E} \backslash\{\ell \sim \delta\} .
$$

We will prove below the following relation.

Lemma 7.1. We have

$$
L=\mathbb{E}_{\nu}\left[e^{-\frac{1}{2}\left\langle\theta, H_{\beta}^{-1} \theta\right\rangle}\right]=\lim _{W_{\ell \delta \downarrow}} \mathbb{E}_{\mu^{W}}\left[e^{-\frac{1}{2}\left\langle\theta, H_{\beta}^{-1} \theta\right\rangle}\right],
$$

where $W_{\ell \delta}$ is the (positive) weight associated to the edge $\ell \sim \delta$.

Step 2. To construct the analog of the additional gamma variable in Sabot et al. $(2017+)$, we select now as special vertex in $V$ the unique vertex $\ell$ connected to $\delta$.

Let us consider the reduced graph consisting of the vertex set $V^{\circ}=V \backslash\{\ell\}$ and edge set $E^{\circ}=E \backslash\{(i \sim \ell): i \in V\}$. In the same way, let $W^{\circ} \in \mathbb{R}^{V \times V}$ be the reduced weight matrix given by $W_{i j}^{\circ}=W_{i j}$ for $i, j \in V$.

With respect to the smaller graph $\mathcal{G}=(V, E)$, the objects $V^{\circ}, E^{\circ}, \ell, V, E, W^{\circ}$, and $U_{V^{\circ}}$ play the same role as $V, E, \delta, \tilde{V}, \tilde{E}, W$, and $U_{V}$, with respect to the larger 
original graph $\tilde{\mathcal{G}}=(\tilde{V}, \tilde{E})$. In particular, we have the following analog of $(2.5)$ :

$$
\begin{aligned}
& \hat{G}^{V^{\circ}, W^{\circ}}: U_{V^{\circ}} \rightarrow \mathbb{R}^{V \times V}, \\
& \hat{G}_{i j}^{V^{\circ}, W^{\circ}}(\tilde{u})= \begin{cases}e^{\tilde{u}_{i}}\left(A_{V^{\circ} V^{\circ}}^{W^{\circ}}(\tilde{u})^{-1}\right)_{i j} e^{\tilde{u}_{j}} & i, j \in V^{\circ}, \\
0 & i=\ell \text { or } j=\ell .\end{cases}
\end{aligned}
$$

Recall that $H_{\beta(u)}^{-1}=\hat{G}_{V V}^{V, W}(u)$ by (2.12). To relate $U_{V}$ and $U_{V^{\circ}}$ we define the shift

$$
\sim: U_{V} \rightarrow U_{V^{\circ}}, \quad u \mapsto \tilde{u}=\left(\tilde{u}_{i}=u_{i}-u_{\ell}\right)_{i \in V} .
$$

Then we have the following relation between $\hat{G}_{V V}^{V, W}(u)$ and $\hat{G}^{V^{\circ}, W^{\circ}}(\tilde{u})$.

Lemma 7.2. The matrices $\hat{G}_{V V}^{V, W}(u)$ and $\hat{G}^{V^{\circ}, W^{\circ}}(\tilde{u})$ satisfy the following relation

$$
\hat{G}_{i j}^{V, W}(u)=\frac{e^{\tilde{u}_{i}+\tilde{u}_{j}}}{W_{\ell \delta} e^{-u_{\ell}}}+\hat{G}_{i j}^{V^{\circ}, W^{\circ}}(\tilde{u}) \quad \text { for all } i, j \in V .
$$

This relation is an analog to the second formula in Proposition 8 of Sabot and Zeng (2015). The proof is given below.

Step 3. Using (7.8), we get

$$
\left\langle\theta, H_{\beta}^{-1} \theta\right\rangle=\left\langle\theta, \hat{G}_{V V}^{V, W}(u) \theta\right\rangle=\left\langle\theta, \hat{G}^{V^{\circ}, W^{\circ}}(\tilde{u}) \theta\right\rangle+\frac{e^{u_{\ell}}}{W_{\ell \delta}}\left\langle\theta, e^{\tilde{u}}\right\rangle^{2} .
$$

Inserting this in (7.5), we obtain

$$
\begin{aligned}
L & =\lim _{W_{\ell \delta \downarrow 0}} \mathbb{E}_{\mu^{W}}\left[e^{\left.-\frac{1}{2}\left\langle\theta, H_{\beta}^{-1} \theta\right\rangle\right]}=\lim _{W_{\ell \delta} \downarrow 0} \mathbb{E}_{\mu W}\left[e^{-\frac{1}{2}\left\langle\theta, \hat{G}^{V^{\circ}, W^{\circ}}(\tilde{u}) \theta\right\rangle-\frac{e^{u} \ell}{2 W_{\ell \delta}}\left\langle\theta, e^{\tilde{u}}\right\rangle^{2}}\right]\right. \\
& =\lim _{W_{\ell \delta \downarrow 0}} \mathbb{E}_{\mu^{W}}\left[e^{-\frac{1}{2}\left\langle\theta, \hat{G}^{V^{\circ}, W^{\circ}}(\tilde{u}) \theta\right\rangle_{\mathbb{E}_{\mu} W}}\left[e^{-\frac{e^{u_{\ell}}}{2 W_{\ell \delta}}\left\langle\theta, e^{\tilde{u}}\right\rangle^{2}} \mid \tilde{u}\right]\right]
\end{aligned}
$$

In the following, we denote the $H^{2 \mid 2}$-measure $\mu^{W}$, defined in (2.4), by $\mu_{\delta}^{W, \tilde{\mathcal{G}}}$, in order to stress the dependence on the graph $\tilde{\mathcal{G}}$ and the reference point $\delta$, which satisfies $u_{\delta}=0$. The conditional expectation is described in the following lemma.

Lemma 7.3. Let $\tilde{u} \in U_{V^{\circ}}$ be defined as in (7.7). We have

$$
\mathbb{E}_{\mu_{\delta}^{W, \tilde{\mathcal{G}}}}\left[e^{-\frac{e^{u_{\ell}}}{2 W_{\ell \delta}}\left\langle\theta, e^{\tilde{u}}\right\rangle^{2}} \mid \tilde{u}\right]=e^{W_{\delta \ell}-\sqrt{W_{\delta \ell}^{2}+\left\langle\theta, e^{\tilde{u}\rangle^{2}}\right.}} .
$$

The proof uses independence of $\tilde{u}$ and $u_{\ell}$ with respect to $\mu_{\delta}^{W, \tilde{\mathcal{G}}}$. It is given below. Now, inserting (7.11) into (7.10), we obtain

$$
\begin{aligned}
L & =\lim _{W_{\ell \delta \downarrow 0}} \mathbb{E}_{\mu_{\delta}^{W, \tilde{\mathcal{G}}}}\left[e^{-\frac{1}{2}\left\langle\theta, \hat{G}^{V^{\circ}, W^{\circ}}(\tilde{u}) \theta\right\rangle} e^{W_{\delta \ell}-\sqrt{W_{\delta \ell}^{2}+\left\langle\theta, e^{\tilde{u}}\right\rangle^{2}}}\right] \\
& =\mathbb{E}_{\mu_{\delta}^{W, \tilde{\mathcal{G}}}}\left[e^{-\frac{1}{2}\left\langle\theta, \hat{G}^{V^{\circ}, W^{\circ}}(\tilde{u}) \theta\right\rangle-\left\langle\theta, e^{\tilde{u}}\right\rangle}\right] .
\end{aligned}
$$

The measure $\mu_{\delta}^{W, \tilde{\mathcal{G}}}$, on the bigger weighted graph $(\tilde{\mathcal{G}}, W)$ with reference point $\delta$, is related to the measure $\mu_{\ell}^{W^{\circ}, \mathcal{G}}$ on the smaller weighted graph $\left(\mathcal{G}, W^{\circ}\right)$ with reference point $\ell$ as follows. The $\mu_{\delta}^{W, \tilde{\mathcal{G}}}$-law of $\tilde{u}=\left(\tilde{u}_{i}=u_{i}-u_{\ell}\right)_{i \in V}$, with $u \in U_{V}$, equals the $\mu_{\ell}^{W^{\circ}, \mathcal{G}}$-law of $u=\left(u_{i}\right)_{i \in V} \in U_{V^{\circ}}$. Hence, applying (2.8) from Theorem 2.1 with $-\theta$ and $\lambda=0$, we get

$$
L=\mathbb{E}_{\mu_{\ell} W^{\circ}, \mathcal{G}}\left[e^{-\frac{1}{2}\left\langle\theta, \hat{G}^{V^{\circ}, W^{\circ}}(u) \theta\right\rangle-\left\langle\theta, e^{u}\right\rangle}\right]=\mathcal{L}^{W^{\circ}}(0) e^{\langle-\theta, 1\rangle}=e^{-\langle\theta, 1\rangle} .
$$


This proves formula (2.13) in the special case $\phi=1$.

Finally, we give the proof of Lemmas 7.1-7.3.

Proof of Lemma \%.1: By Proposition 1 of Sabot et al. (2017+), the Laplace transform of $\beta=\left(\beta_{i}\right)_{i \in V}$ with respect to $\nu$ is given by

$$
\mathbb{E}_{\nu}\left[e^{-\langle\lambda, \beta\rangle}\right]=\prod_{(i \sim j) \in E} e^{W_{i j}\left(1-\sqrt{1+\lambda_{i}} \sqrt{1+\lambda_{j}}\right)} \prod_{i \in V} \frac{1}{\sqrt{1+\lambda_{i}}}
$$

for $\lambda \in(-1, \infty)^{V}$. Comparing with formula (2.9) from Theorem 2.1, we find for these $\lambda$

$$
\mathbb{E}_{\nu}\left[e^{-\langle\lambda, \beta\rangle}\right]=\mathbb{E}_{\mu^{W}}\left[e^{-\langle\lambda, \beta\rangle}\right] e^{-W_{\ell \delta}\left(1-\sqrt{1+\lambda_{\ell}}\right)}
$$

with the $H^{2 \mid 2}$ measure $\mu^{W}$ defined in (2.4). To see this, one may extend $\lambda$ by the additional value $\lambda_{\delta}=0$.

Both sides of (7.15) are complex analytic functions of $\lambda \in((-1, \infty)+i \mathbb{R})^{V}$. The square root is understood as its principal branch, i.e. $\sqrt{r^{2} e^{2 i \varphi}}=r e^{i \varphi}$ for $r>0$, $-\pi<\varphi<\pi$. Although equation (7.15) was derived for real $\lambda \in(-1, \infty)^{V}$ only, the identity theorem for holomorphic functions implies that it holds also for complex $\lambda \in((-1, \infty)+i \mathbb{R})^{V}$. The identity (7.15) holds for any value $W_{\ell \delta}>0$. Hence, for all $\lambda \in((-1, \infty)+i \mathbb{R})^{V}$, one has

$$
\mathbb{E}_{\nu}\left[e^{-\langle\lambda, \beta\rangle}\right]=\lim _{W_{\ell \delta \downarrow 0}} \mathbb{E}_{\mu W}\left[e^{-\langle\lambda, \beta\rangle}\right],
$$

where in the last limit $W_{i j}$ is kept fixed unless $\{i, j\}=\{\ell, \delta\}$. In particular, taking imaginary $\lambda \in(i \mathbb{R})^{V}$, equation (7.16) shows a pointwise convergence of Fourier transforms. We conclude that $\operatorname{law}_{\mu^{W}}(\beta)$ converges weakly to $\operatorname{law}_{\nu}(\beta)$ as $W_{\ell \delta} \downarrow 0$. Since $H_{\beta}$ is positive definite, $\left\langle\theta, H_{\beta}^{-1} \theta\right\rangle>0$. Note that $1_{\left\{H_{\beta}>0\right\}} \exp \left(-\frac{1}{2}\left\langle\theta, H_{\beta}^{-1} \theta\right\rangle\right)$ is a bounded function of $\beta \in \mathbb{R}^{V}$ and its set of discontinuities has $\nu$-measure 0 . Consequently, using weak convergence, the result follows.

Proof of Lemma 7.2: We write $H_{b}^{W}$ rather than $H_{b}$ because we are working with varying weights $W$. Remember that $\hat{G}_{V V}^{V, W}(u)=\left(H_{\beta^{V}(u)}^{W}\right)^{-1}$. By using the partition $V=V^{\circ} \cup\{\ell\}$, we can write

$$
H_{\beta^{V}(u)}^{W}=\left(\begin{array}{cc}
2 \beta_{\ell}(u) & -W_{\ell V^{\circ}} \\
-W_{V^{\circ} \ell} & M
\end{array}\right),
$$

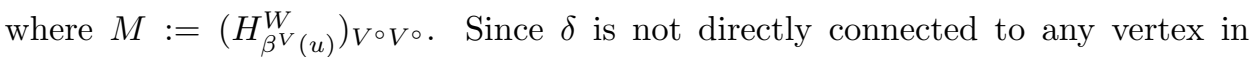
$V^{\circ}$, we have $\beta^{W^{\circ}}(\tilde{u})=\beta^{W}(u)_{V^{\circ}}$. Hence $\left(H_{\beta^{\circ}(\tilde{u})}^{W^{\circ}}\right)_{V^{\circ} V^{\circ}}=\left(H_{\beta^{V}(u)}^{W}\right)_{V^{\circ} V^{\circ}}$, and we conclude

$$
\hat{G}_{V^{\circ} V^{\circ}}^{V^{\circ}}(\tilde{u})=M^{-1}
$$

We can write $\left(H_{\beta^{V}(u)}^{W}\right)^{-1}$ using the following block-matrix inversion formula

$$
\left(\begin{array}{ll}
A & C \\
D & B
\end{array}\right)^{-1}=\left(\begin{array}{cc}
b^{-1} & -b^{-1} C B^{-1} \\
-B^{-1} D b^{-1} & B^{-1}+B^{-1} D b^{-1} C B^{-1}
\end{array}\right)
$$


with $b=A-C B^{-1} D$, which holds if $B$ and $b$ are invertible. In our case $b=$ $2 \beta_{\ell}(u)-W_{\ell V^{\circ}} M^{-1} W_{V^{\circ} \ell}$ is a scalar and

$$
2 \beta_{\ell}(u)=W_{\ell \delta} e^{-u_{\ell}}+\sum_{j \in V^{\circ}} W_{\ell j} e^{\tilde{u}_{j}}=W_{\ell \delta} e^{-u_{\ell}}+W_{\ell V^{\circ}} e_{V^{\circ}}^{\tilde{u}}
$$

with $e_{V^{\circ}}^{\tilde{u}}=\left(e^{\tilde{u}_{i}}\right)_{i \in V^{\circ}}$. Hence

$$
\begin{aligned}
\left(H_{\beta^{V}(u)}^{W}\right)^{-1} & =\frac{1}{b}\left(\begin{array}{cc}
1 & W_{\ell V^{\circ}} M^{-1} \\
M^{-1} W_{V \circ \ell} & M^{-1} W_{V^{\circ} \ell} W_{\ell V} \circ M^{-1}
\end{array}\right)+\left(\begin{array}{cc}
0 & 0 \\
0 & M^{-1}
\end{array}\right) \\
& =\frac{1}{b}\left(\begin{array}{c}
1 \\
M^{-1} W_{V \circ \ell}
\end{array}\right)\left(\begin{array}{c}
1 \\
M^{-1} W_{V \circ \ell}
\end{array}\right)^{t}+\hat{G}^{V^{\circ}, W^{\circ}}(\tilde{u}) .
\end{aligned}
$$

Now recall that, by (3.19), we have $H_{\beta(u)}^{W} e_{V}^{u}=W_{V \delta}$, for all $u \in U_{V}$. Applying the same identity to the smaller graph $(V, E)$ with reference point $\ell$ we obtain $H_{\beta(\tilde{u})}^{W^{\circ}} e_{V^{\circ}}^{\tilde{u}}=W_{V^{\circ} \ell}$, for all $\tilde{u} \in U_{V^{\circ}}$. We obtain

$$
M^{-1} W_{V^{\circ \ell}}=e_{V^{\circ}}^{\tilde{u}}, \quad\left(\begin{array}{c}
1 \\
M^{-1} W_{V^{\circ} \ell}
\end{array}\right)=e_{V}^{\tilde{u}} .
$$

Furthermore, $b=W_{\ell \delta} e^{-u_{\ell}}$ and (7.21) yields the claim written in matrix form:

$$
\hat{G}_{V V}^{V, W}(u)=e_{V}^{\tilde{u}} \frac{1}{W_{\ell \delta} e^{-u_{\ell}}}\left(e_{V}^{\tilde{u}}\right)^{t}+\hat{G}^{V^{\circ}, W^{\circ}}(\tilde{u}) .
$$

Proof of Lemma 7.3: Let $\Gamma$ denote the graph consisting only of the two vertices $\ell$ and $\delta$ and the edge $\ell \sim \delta$ with weight $W_{\ell \delta}$ connecting them. Using Lemma A.1 of Disertori et al. (2016), the laws of $u_{\ell}$ with respect to $\mu_{\delta}^{W, \tilde{\mathcal{G}}}$ and $\mu_{\delta}^{W_{\ell \delta}, \Gamma}$ coincide and the gradient variables $\tilde{u}$ are independent of $u_{\ell}$ with respect to $\mu_{\delta}^{W, \tilde{\mathcal{G}}}$. Thus, abbreviating $C(\tilde{u})=\left\langle\theta, e^{\tilde{u}}\right\rangle^{2} /\left(2 W_{\ell \delta}\right)$, we get

$$
\mathbb{E}_{\mu_{\delta}^{W, \tilde{\mathcal{G}}}}\left[e^{-\frac{e^{u_{\ell}}}{2 W_{\ell \delta}}\left\langle\theta, e^{\tilde{u}}\right\rangle^{2}} \mid \tilde{u}\right]=\left.\mathbb{E}_{\mu_{\delta}^{W, \tilde{\mathcal{G}}}}\left[e^{-c e^{u_{\ell}}}\right]\right|_{c=C(\tilde{u})}=\left.\mathbb{E}_{\mu_{\delta}^{W_{\ell \delta}, \Gamma}}\left[e^{-c e^{u_{\ell}}}\right]\right|_{c=C(\tilde{u})} .
$$

In order to compute the last expectation, we exchange the role of $\delta$ and $\ell$ using $\ell$ as new reference point. The $\mu_{\delta}^{W_{\ell \delta}, \Gamma}$-law of $u_{\ell}-u_{\delta}$ has the Radon-Nikodym derivative $e^{u_{\delta}-u_{\ell}}$ with respect to the $\mu_{\ell}^{W_{\ell \delta}, \Gamma}$-law of the same function $u_{\ell}-u_{\delta}$. To see this, note that $t=u_{\ell}-u_{\delta}$ has distribution

$$
\sqrt{\frac{W_{\ell \delta}}{2 \pi}} e^{-W_{\ell \delta}(\cosh t-1)} e^{-\frac{t}{2}} d t
$$

with respect to $\mu_{\delta}^{W_{\ell \delta}, \Gamma}$. Hence, for any $c>0$, we obtain

$$
\begin{aligned}
\mathbb{E}_{\mu_{\delta} W_{\ell \delta}, \Gamma}\left[e^{-c e^{u_{\ell}}}\right] & =\mathbb{E}_{\mu_{\delta}^{W_{\ell \delta}, \Gamma}}\left[e^{-c e^{u_{\ell}-u_{\delta}}}\right] \\
& =\mathbb{E}_{\mu_{\ell}^{W_{\ell \delta}, \Gamma}}\left[e^{u_{\delta}-u_{\ell}} e^{-c e^{u_{\ell}-u_{\delta}}}\right]=\mathbb{E}_{\mu_{\ell} W_{\ell \delta}, \Gamma}\left[e^{u_{\delta}} e^{-c e^{-u_{\delta}}}\right] .
\end{aligned}
$$


Note that for the weighted graph $\left(\Gamma, W_{\ell \delta}\right)$ with reference $\ell$, we have $\beta_{\delta}=\beta_{\delta}^{W_{\ell \delta}}=$ $\frac{1}{2} W_{\ell \delta} e^{-u_{\delta}}$. Thus, abbreviating $\lambda_{\delta}=2 c / W_{\ell \delta}$, we obtain from formula (3.15) in Example 3.4

$$
\begin{aligned}
\mathbb{E}_{\mu_{\ell} W_{\ell \delta}, \Gamma}\left[e^{u_{\delta}} e^{-c e^{-u_{\delta}}}\right] & =\mathbb{E}_{\mu_{\ell}^{W_{\ell \delta}, \Gamma}}\left[e^{u_{\delta}} e^{-\lambda_{\delta} \beta_{\delta}}\right] \\
& =\mathcal{L}^{W_{\ell \delta}}\left(\lambda_{\delta}\right) \sqrt{1+\lambda_{\delta}}=e^{W_{\ell \delta}\left(1-\sqrt{1+\lambda_{\delta}}\right)}
\end{aligned}
$$

in the last equality we used (3.11) to calculate $\mathcal{L}^{W_{\ell \delta}}\left(\lambda_{\delta}\right)$. Summarizing, this shows

$$
\mathbb{E}_{\mu_{\delta}^{W, \tilde{\mathcal{G}}}}\left[e^{-\frac{e^{u_{\ell}}}{2 W_{\ell \delta}}\left\langle\theta, e^{\tilde{u}}\right\rangle^{2}} \mid \tilde{u}\right]=e^{W_{\ell \delta}\left[1-\sqrt{1+2 C(\tilde{u}) W_{\ell \delta}^{-1}}\right]}=e^{W_{\ell \delta}-\sqrt{W_{\ell \delta}^{2}+\left\langle\theta, e^{\tilde{u}}\right\rangle^{2}}} .
$$

7.2. General case. We deduce the general case of (2.13) from the special case $\phi=1$ using a scaling argument. In this part of the proof, we write again $H_{b}^{W}$ rather than $H_{b}$. Let $\phi, \theta \in(0, \infty)^{V}$. We consider the change of variables $b_{i}^{\prime}=\phi_{i}^{2} b_{i}$ for all $i \in V$ and the rescaled weights $W_{i j}^{\prime}=\phi_{i} W_{i j} \phi_{j}$. Denoting by $\operatorname{diag} \phi \in \mathbb{R}^{V \times V}$ the diagonal matrix with diagonal entries $\phi_{i}, i \in V$, we have for $i, j \in V$ and $b \in \mathbb{R}^{V}$

$$
\left(\operatorname{diag} \phi H_{b}^{W} \operatorname{diag} \phi\right)_{i j}=2 \phi_{i} b_{i} \phi_{j} \delta_{i j}-\phi_{i} W_{i j} \phi_{j}=2 b_{i}^{\prime} \delta_{i j}-W_{i j}^{\prime}=\left(H_{b^{\prime}}^{W^{\prime}}\right)_{i j} .
$$

Thus, $\operatorname{diag} \phi H_{b}^{W} \operatorname{diag} \phi=H_{b^{\prime}}^{W^{\prime}}$. From this, we deduce

$$
\left(H_{b}^{W}\right)^{-1}=\operatorname{diag} \phi\left(H_{b^{\prime}}^{W^{\prime}}\right)^{-1} \operatorname{diag} \phi \quad \text { and } \quad \frac{1}{\sqrt{\operatorname{det} H_{b}^{W}}}=\frac{\prod_{i \in V} \phi_{i}}{\sqrt{\operatorname{det} H_{b^{\prime}}^{W^{\prime}}}} .
$$

Furthermore, $H_{b}^{W}>0$ if and only if $H_{b^{\prime}}^{W^{\prime}}>0$. Changing variables from $b$ to $b^{\prime}$ we get the Jacobi determinant $\left|d b / d b^{\prime}\right|=\left(\prod_{i \in V} \phi_{i}\right)^{-2}$. Abbreviating $\theta \phi=\left(\theta_{i} \phi_{i}\right)_{i \in V}$, we conclude

$$
\begin{aligned}
& \int_{\left\{b \in \mathbb{R}^{V}: H_{b}^{W}>0\right\}} \frac{e^{-\frac{1}{2}\left(\left\langle\phi, H_{b}^{W} \phi\right\rangle+\left\langle\theta,\left(H_{b}^{W}\right)^{-1} \theta\right\rangle\right)}}{\sqrt{\operatorname{det} H_{b}^{W}}} d b \\
= & \int_{\left\{b^{\prime} \in \mathbb{R}^{V}: H_{b^{\prime}}^{W^{\prime}}>0\right\}} \frac{e^{-\frac{1}{2}\left(\left\langle 1, H_{b^{\prime}}^{W^{\prime}} 1\right\rangle+\left\langle\theta \phi,\left(H_{b^{\prime}}^{W^{\prime}}\right)^{-1} \theta \phi\right\rangle\right)}}{\sqrt{\operatorname{det} H_{b^{\prime}}^{W^{\prime}}} \prod_{i \in V} \phi_{i}} d b^{\prime}=\left(\frac{\pi}{2}\right)^{\frac{|V|}{2}} \frac{e^{-\langle 1, \phi \theta\rangle}}{\prod_{i \in V} \phi_{i}},
\end{aligned}
$$

where we used (2.13) for the special case $\phi=1$ treated in Section 7.1 above. Since $\langle 1, \phi \theta\rangle=\langle\phi, \theta\rangle$ the claim (2.13) follows.

\section{Appendix A. Geometric intuition.}

The supersymmetric hyperbolic nonlinear sigma model lives over a supermanifold $H^{2 \mid 2}$ extending the hyperboloid

$$
H^{2}=\left\{v=(a, b, c) \in \mathbb{R}^{3}: c>0,\langle v, v\rangle_{h}=-1\right\},
$$

where the hyperbolic inner product $\langle\cdot, \cdot\rangle_{h}$ is defined by

$$
\langle v, \tilde{v}\rangle_{h}=a \tilde{a}+b \tilde{b}-c \tilde{c}
$$

for $v=(a, b, c), \tilde{v}=(\tilde{a}, \tilde{b}, \tilde{c}) \in \mathbb{R}^{3}$. The restriction of $\langle\cdot, \cdot\rangle_{h}$ to the tangential spaces of $H^{2}$ is positive definite although the hyperbolic inner product itself is indefinite. 
Consequently, this restriction gives a Riemannian metric on $H^{2}$. The corresponding geodesic distance is called hyperbolic distance and is given by

$$
d_{h}(v, \tilde{v})=\operatorname{arcosh}\left(-\langle v, \tilde{v}\rangle_{h}\right)=\operatorname{arcosh}\left(1+\frac{1}{2}\langle v-\tilde{v}, v-\tilde{v}\rangle_{h}\right)
$$

for $v, \tilde{v} \in H^{2}$. The right-hand side is well-defined because of $\langle v, \tilde{v}\rangle_{h} \leq-1$. The hyperbolic distance $d_{h}$ is a metric on $H^{2}$. In particular, $d_{h}$ satisfies the triangle inequality. The supersymmetric extension $H^{2 \mid 2}$ is obtained by replacing $v \in H^{2}$ by $\boldsymbol{v}=(x, y, z, \xi, \eta)$ with even elements $x, y, z$ and odd elements $\xi, \eta$ and the hyperbolic inner product by

$$
\langle\boldsymbol{v}, \tilde{\boldsymbol{v}}\rangle_{s h}=x \tilde{x}+y \tilde{y}-z \tilde{z}+\xi \tilde{\eta}-\eta \tilde{\xi} .
$$

Note that the constraint $\langle\boldsymbol{v}, \boldsymbol{v}\rangle_{s h}=-1$ implies $z^{2}=1+x^{2}+y^{2}+2 \xi \eta$; cf. formula (5.7). In horospherical coordinates $H^{2}$ is parametrized by

$$
\begin{aligned}
& H^{2}=\{(a(u, s), b(u, s), c(u, s)): u, s \in \mathbb{R}\}, \quad \text { with } \\
& a(u, s)=\sinh u-\frac{1}{2} s^{2} e^{u}, \quad b(u, s)=s e^{u}, \quad c(u, s)=\cosh u+\frac{1}{2} s^{2} e^{u} .
\end{aligned}
$$

Therefore equation (5.6) yields a supersymmetric extension of these horospherical coordinates. The action $\mathcal{S}_{\text {cart }}$ defined in (5.9) can be now written as

$$
\mathcal{S}_{\text {cart }}=-\frac{1}{2} \sum_{(j \sim k) \in \tilde{E}} W_{j k}\left\langle\boldsymbol{v}_{j}-\boldsymbol{v}_{k}, \boldsymbol{v}_{j}-\boldsymbol{v}_{k}\right\rangle_{s h}=\sum_{(j \sim k) \in \tilde{E}} W_{j k}\left(1+\left\langle\boldsymbol{v}_{j}, \boldsymbol{v}_{k}\right\rangle_{s h}\right) .
$$

\section{Appendix B. Some useful results.}

Lemma B.1. The superfunction $\mathcal{S}_{\text {cart }}$ fulfills $e^{\mathcal{S}_{\text {cart }}} \in \mathbb{S}_{\text {susy }}\left(\Omega_{V}, \xi, \eta\right)$.

Proof: For any superfunction $f_{\text {cart }}(x, y, \xi, \eta)$ we define its body as the first term in the expansion (5.11): $\operatorname{body}\left(f_{\text {cart }}\right)=f_{\emptyset \emptyset}: \Omega_{V} \rightarrow \mathbb{C}$. Note that all coefficients in the expansion (5.11) of $e^{\mathcal{S}_{\text {cart }}}$, together with all their derivatives of any order in $x$ and $y$, are of the form $p \cdot e^{\operatorname{body}\left(\mathcal{S}_{\text {cart }}\right)}$, where $p: \Omega_{V} \rightarrow \mathbb{R}$ are algebraic functions of $x$ and $y$, nowhere singular. Therefore, it is enough to show that for all $(x, y) \in \Omega_{V}$

$$
-\operatorname{body}\left(\mathcal{S}_{\text {cart }}\right)(x, y) \geq \frac{1}{2} c(W) \max _{i \in \tilde{V}}\left\|\left(x_{i}, y_{i}\right)\right\|^{\frac{1}{r}}-c(W)
$$

holds with a constant $c(W)>0$ and $r=r(\tilde{\mathcal{G}}, \rho)$ being the maximum of all graph distances in $\tilde{\mathcal{G}}$ from vertices $i \in V$ to the reference vertex $\rho$. Observe that

$$
\begin{aligned}
& -\operatorname{body}\left(\mathcal{S}_{\text {cart }}\right)(x, y)=\frac{1}{2} \sum_{(j \sim k) \in \tilde{E}} W_{j k}\left\langle v_{j}-v_{k}, v_{j}-v_{k}\right\rangle_{h} \\
= & \sum_{(i \sim j) \in \tilde{E}} W_{i j}\left(-1-\left\langle v_{i}, v_{j}\right\rangle_{h}\right)=\sum_{(j \sim k) \in \tilde{E}} W_{j k}\left(\cosh d_{h}\left(v_{j}, v_{k}\right)-1\right),
\end{aligned}
$$

where $v_{i}=\left(x_{i}, y_{i}, z_{i}^{\prime}\right)$ and $z_{i}^{\prime}=\sqrt{1+x_{i}^{2}+y_{i}^{2}}=\operatorname{body}\left(z_{i}\right)$ for $i \in V$.

Let $o=(0,0,1) \in H^{2}$. In particular, $v_{\rho}=o$. Given a vertex $i \in V$, we take a path $\pi=\left(i_{0}=\rho, i_{1}, \ldots, i_{n}=i\right)$ in $\tilde{\mathcal{G}}$ from $\rho$ to $i$ with length $n \leq r$, where $r$ is the 
maximum graph distance defined below equation (B.1). By the triangle inequality for $d_{h}$, we know

$$
d_{h}\left(v_{i}, o\right) \leq \sum_{m=1}^{n} d_{h}\left(v_{i_{m-1}}, v_{i_{m}}\right) \leq r \max \left\{d_{h}\left(v_{j}, v_{k}\right):(j \sim k) \in \tilde{E}\right\} .
$$

Using the fact $\cosh (r a) \leq(2 \cosh a)^{r}$ for $a \geq 0$ and the definition (A.3) of the hyperbolic distance $d_{h}(v, \tilde{v})$, we can write

$$
\begin{aligned}
\left\|\left(x_{i}, y_{i}\right)\right\| & \leq z_{i}^{\prime}=-\left\langle v_{i}, o\right\rangle_{h}=\cosh d_{h}\left(v_{i}, o\right) \\
& \leq \max _{(j \sim k) \in \tilde{E}} \cosh \left(r d_{h}\left(v_{j}, v_{k}\right)\right) \leq\left(2 \max _{(j \sim k) \in \tilde{E}} \cosh d_{h}\left(v_{j}, v_{k}\right)\right)^{r} .
\end{aligned}
$$

Taking the $r$-th root and the maximum over $i$ yields

$$
\max _{(j \sim k) \in \tilde{E}} \cosh d_{h}\left(v_{j}, v_{k}\right) \geq \frac{1}{2} \max _{i \in \tilde{V}}\left\|\left(x_{i}, y_{i}\right)\right\|^{\frac{1}{r}} .
$$

Inserting these in $\operatorname{body}\left(\mathcal{S}_{\text {cart }}\right)$, cf. (B.2), we obtain

$$
\begin{aligned}
-\operatorname{body}\left(\mathcal{S}_{\text {cart }}\right) & \geq c(W)\left(\max _{(j \sim k) \in \tilde{E}} \cosh d_{h}\left(v_{j}, v_{k}\right)-1\right) \\
& \geq \frac{1}{2} c(W) \max _{i \in \tilde{V}}\left\|\left(x_{i}, y_{i}\right)\right\|^{\frac{1}{r}}-c(W)
\end{aligned}
$$

with the constant $c(W)=\min _{(j \sim k) \in \tilde{E}} W_{j k}>0$. This concludes the proof.

Proof of Lemma 5.1: We factorize the supertransformation in (5.6) from the cartesian coordinates $(x, y, \xi, \eta)$ to horospherical coordinates $(u, s, \bar{\psi}, \psi)$ in a composition of four supertransformations as follows.

(1) The first step consists of the substitution $\xi_{i}=\tilde{\xi}_{i} \sqrt{x_{i}+z_{i}^{\prime}}, \eta_{i}=\tilde{\eta}_{i} \sqrt{x_{i}+z_{i}^{\prime}}$, where we define $z_{i}^{\prime}=z_{i}^{\prime}(x, y)=\sqrt{1+x_{i}^{2}+y_{i}^{2}}$.

Recall $z_{i}(x, y, \xi, \eta)=\sqrt{1+x_{i}^{2}+y_{i}^{2}+2 \xi_{i} \eta_{i}}$. We set

$$
f_{1}(x, y, \tilde{\xi}, \tilde{\eta})=\left(e^{\mathcal{S}_{\text {cart }}} f_{\text {cart }} \prod_{j \in V} \frac{1}{z_{j}}\right)\left(\left(x_{i}, y_{i}, \tilde{\xi}_{i} \sqrt{x_{i}+z_{i}^{\prime}}, \tilde{\eta}_{i} \sqrt{x_{i}+z_{i}^{\prime}}\right)_{i \in V}\right) .
$$

Note that when $e^{\mathcal{S}_{\text {cart }}} f_{\text {cart }} \in \mathbb{S}_{\text {susy }}\left(\Omega_{V}, \xi, \eta\right)$, then $f_{1} \in \mathbb{S}_{\text {susy }}\left(\Omega_{V}, \tilde{\xi}, \tilde{\eta}\right)$. Moreover, since $\xi_{i}^{2}=0=\eta_{i}^{2}$, we have $\tilde{\xi}_{i} \sqrt{x_{i}+z_{i}^{\prime}}=\tilde{\xi}_{i} \sqrt{x_{i}+z_{i}}$ and $\tilde{\eta}_{i} \sqrt{x_{i}+z_{i}^{\prime}}=\tilde{\eta}_{i} \sqrt{x_{i}+z_{i}}$. In the new coordinates, $z_{i}$ becomes

$$
z_{i}=\sqrt{1+x_{i}^{2}+y_{i}^{2}+2\left(x_{i}+z_{i}^{\prime}\right) \tilde{\xi}_{i} \tilde{\eta}_{i}} .
$$

Under the first coordinate change, the Grassmann derivatives in $D \mu_{\text {cart }}^{W}(5.8)$ are transformed as $\prod_{j \in V} \partial_{\xi_{j}} \partial_{\eta_{j}}=\prod_{j \in V} \frac{1}{x_{j}+z_{j}^{\prime}} \partial_{\tilde{\xi}_{j}} \partial_{\tilde{\eta}_{j}}$. Hence,

$$
\mathbb{E}_{D \mu_{\text {cart }}^{W}}^{W}\left[f_{\text {cart }}\right]=\int_{\Omega_{V}}\left(\prod_{j \in V} \frac{d x_{j} d y_{j}}{2 \pi} \partial_{\tilde{\xi}_{j}} \partial_{\tilde{\eta}_{j}}\right)\left(f_{1}(x, y, \tilde{\xi}, \tilde{\eta}) \prod_{j \in V} \frac{1}{x_{j}+z_{j}^{\prime}}\right)
$$


Observe that in the original coordinates, $z_{j}=z_{j}^{\prime}+\frac{1}{z_{j}^{\prime}} \xi_{j} \eta_{j}, z_{j} \xi_{j} \eta_{j}=z_{j}^{\prime} \xi_{j} \eta_{j}$, and $z_{j}^{\prime} \tilde{\xi}_{j} \tilde{\eta}_{j}=z_{j} \tilde{\xi}_{j} \tilde{\eta}_{j}$. Using these identities, we obtain

$$
\begin{aligned}
z_{j}\left(x_{j}+z_{j}^{\prime}\right) & =z_{j}\left(x_{j}+z_{j}-\frac{1}{z_{j}^{\prime}} \xi_{j} \eta_{j}\right)=z_{j}\left(x_{j}+z_{j}\right)-\xi_{j} \eta_{j} \\
& =\left(x_{j}+z_{j}\right)\left(z_{j}-\tilde{\xi}_{j} \tilde{\eta}_{j}\right) .
\end{aligned}
$$

We abbreviate

$$
\begin{aligned}
& F_{1}(x, y, \tilde{\xi}, \tilde{\eta})=f_{1}(x, y, \tilde{\xi}, \tilde{\eta}) \prod_{j \in V} \frac{1}{x_{j}+z_{j}^{\prime}} \\
= & \left(e^{\mathcal{S}_{\text {cart }}} f_{\text {cart }}\right)\left(\left(x_{i}, y_{i}, \tilde{\xi}_{i} \sqrt{x_{i}+z_{i}^{\prime}}, \tilde{\eta}_{i} \sqrt{x_{i}+z_{i}^{\prime}}\right)_{i \in V}\right) \prod_{j \in V} \frac{1}{\left(x_{j}+z_{j}\right)\left(z_{j}-\tilde{\xi}_{j} \tilde{\eta}_{j}\right)} .
\end{aligned}
$$

Summarizing, we rewrite (B.9) as follows:

$$
\mathbb{E}_{D \mu_{\text {cart }}^{W}}^{W}\left[f_{\text {cart }}\right]=\int_{\Omega_{V}}\left(\prod_{j \in V} \frac{d x_{j} d y_{j}}{2 \pi} \partial_{\tilde{\xi}_{j}} \partial_{\tilde{\eta}_{j}}\right) F_{1}(x, y, \tilde{\xi}, \tilde{\eta}) .
$$

(2) The next transformation will substitute real variables by even elements in a Grassmann algebra. This can be explained as the following automorphism $f \mapsto$ $g:=\left.f\right|_{x=\tilde{x}-\tilde{\xi} \tilde{\eta}}$ of the space of superfunctions $\mathbb{S}_{\text {susy }}\left(\Omega_{V}, \xi, \eta\right)$ :

$$
\begin{aligned}
g(\tilde{x}, y, \tilde{\xi}, \tilde{\eta}) & :=f\left(\left(\tilde{x}_{i}-\tilde{\xi}_{i} \tilde{\eta}_{i}\right)_{i \in V}, y, \tilde{\xi}, \tilde{\eta}\right)=\sum_{a \in\{0,1\}^{V}} D_{\tilde{x}}^{a} f(\tilde{x}, y, \tilde{\xi}, \tilde{\eta})(-\tilde{\xi} \tilde{\eta})^{a} \\
& =f(\tilde{x}, y, \tilde{\xi}, \tilde{\eta})+\sum_{\substack{a \in\{0,1\}^{V} \\
a \neq 0}} D_{\tilde{x}}^{a} f(\tilde{x}, y, \tilde{\xi}, \tilde{\eta})(-\tilde{\xi} \tilde{\eta})^{a}
\end{aligned}
$$

The fundamental theorem of calculus yields

$$
\int_{\Omega_{V}}\left(\prod_{j \in V} \frac{d \tilde{x}_{j} d y_{j}}{2 \pi} \partial_{\tilde{\xi}_{j}} \partial_{\tilde{\eta}_{j}}\right) g(\tilde{x}, y, \tilde{\xi}, \tilde{\eta})=\int_{\Omega_{V}}\left(\prod_{j \in V} \frac{d x_{j} d y_{j}}{2 \pi} \partial_{\tilde{\xi}_{j}} \partial_{\tilde{\eta}_{j}}\right) f(x, y, \tilde{\xi}, \tilde{\eta}) .
$$

Note that this formula contains no boundary terms because all coefficients of $f$ and $g$ are Schwartz functions. Using this automorphism, we define

$$
F_{2}(\tilde{x}, y, \tilde{\xi}, \tilde{\eta})=F_{1}(\tilde{x}-\tilde{\xi} \tilde{\eta}, y, \tilde{\xi}, \tilde{\eta}) \in \mathbb{S}_{\text {susy }}\left(\Omega_{V}, \tilde{\xi}, \tilde{\eta}\right) .
$$

In the new coordinates, with the abbreviation $\tilde{z}_{i}^{\prime}=\sqrt{1+\tilde{x}_{i}^{2}+y_{i}^{2}}$, the variable $z_{i}$ becomes

$$
\left.z_{i}\right|_{x=\tilde{x}-\tilde{\xi} \tilde{\eta}}=\sqrt{1+\tilde{x}_{i}^{2}+y_{i}^{2}+2 \tilde{z}_{i}^{\prime} \tilde{\xi}_{i} \tilde{\eta}_{i}}
$$

Then, equation (B.12) becomes

$$
\mathbb{E}_{D \mu_{\text {cart }}^{W}}\left[f_{\text {cart }}\right]=\int_{\Omega_{V}}\left(\prod_{j \in V} \frac{d \tilde{x}_{j} d y_{j}}{2 \pi} \partial_{\tilde{\xi}_{j}} \partial_{\tilde{\eta}_{j}}\right) F_{2}(\tilde{x}, y, \tilde{\xi}, \tilde{\eta}) .
$$

(3) Setting now $\tilde{x}_{i}=\sinh u_{i}-\frac{1}{2} e^{u_{i}} s_{i}^{2}, y_{i}=s_{i} e^{u_{i}}$, we define

$$
F_{3}(u, s, \tilde{\xi}, \tilde{\eta})=F_{2}\left(\left(\sinh u_{i}-\frac{1}{2} e^{u_{i}} s_{i}^{2}, s_{i} e^{u_{i}}, \tilde{\xi}_{i}, \tilde{\eta}_{i}\right)_{i \in V}\right)
$$


In the new coordinates, $z_{i}$ becomes

$$
z_{i}=\cosh u_{i}+\frac{1}{2} e^{u_{i}} s_{i}^{2}+\tilde{\xi}_{i} \tilde{\eta}_{i}
$$

The Jacobian of the third transformation is given by

$$
\begin{aligned}
\frac{\partial(\tilde{x}, y)}{\partial(u, s)} & =\prod_{i \in V}\left|\begin{array}{cc}
\cosh u_{i}-\frac{1}{2} e^{u_{i}} s_{i}^{2} & -s_{i} e^{u_{i}} \\
s_{i} e^{u_{i}} & e^{u_{i}}
\end{array}\right|=\prod_{i \in V} e^{u_{i}}\left(\cosh u_{i}+\frac{1}{2} e^{u_{i}} s_{i}^{2}\right) \\
& =\prod_{i \in V} e^{u_{i}}\left(z_{i}-\tilde{\xi}_{i} \tilde{\eta}_{i}\right) .
\end{aligned}
$$

Thus with $z_{i}$ as in (B.19), equation (B.17) becomes

$$
\begin{aligned}
& \mathbb{E}_{D \mu_{\text {cart }}^{W}}\left[f_{\text {cart }}\right] \\
= & \int_{\Omega_{V}}\left(\prod_{j \in V} \frac{d u_{j} d s_{j}}{2 \pi} \partial_{\tilde{\xi}_{j}} \partial_{\tilde{\eta}_{j}}\right)\left(F_{3}(u, s, \tilde{\xi}, \tilde{\eta}) \prod_{i \in V} e^{u_{i}}\left(z_{i}-\tilde{\xi}_{i} \tilde{\eta}_{i}\right)\right) .
\end{aligned}
$$

(4) Finally, we set $\tilde{\xi}_{i}=e^{\frac{u_{i}}{2}} \bar{\psi}_{i}, \tilde{\eta}_{i}=e^{\frac{u_{i}}{2}} \psi_{i}$ for $i \in V$. In the new coordinates, $z_{i}$ becomes

$$
z_{i}=\cosh u_{i}+\frac{1}{2} e^{u_{i}} s_{i}^{2}+e^{u_{i}} \bar{\psi}_{i} \psi_{i}
$$

We have $\partial_{\tilde{\xi}_{j}}=e^{-\frac{u_{j}}{2}} \partial_{\bar{\psi}_{j}}, \partial_{\tilde{\eta}_{j}}=e^{-\frac{u_{j}}{2}} \partial_{\psi_{j}}$. Hence, the fourth transformation yields

$$
\prod_{j \in V} \partial_{\tilde{\xi}_{j}} \partial_{\tilde{\eta}_{j}}=\prod_{j \in V} e^{-u_{j}} \partial_{\bar{\psi}_{j}} \partial_{\psi_{j}}
$$

Observe that the term $x_{j}+z_{j}$ from (B.11) is transformed under the coordinate changes as follows:

$$
\begin{aligned}
x_{j}+z_{j} & =\tilde{x}_{j}-\tilde{\xi}_{j} \tilde{\eta}_{j}+z_{j}=\sinh u_{j}-\frac{1}{2} e^{u_{j}} s_{j}^{2}-\tilde{\xi}_{j} \tilde{\eta}_{j}+\cosh u_{j}+\frac{1}{2} e^{u_{j}} s_{j}^{2}+\tilde{\xi}_{j} \tilde{\eta}_{j} \\
& =\sinh u_{j}+\cosh u_{j}=e^{u_{j}} .
\end{aligned}
$$

The composition of the above four coordinate changes gives indeed the supertransformation in (5.6). Inserting all these results in (B.21), we conclude

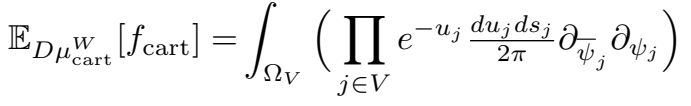

$$
\begin{aligned}
& \left(e^{\mathcal{S}_{\text {cart }}} f_{\text {cart }}\right)(x(u, s, \bar{\psi}, \psi), y(u, s, \bar{\psi}, \psi), \xi(u, s, \bar{\psi}, \psi), \eta(u, s, \bar{\psi}, \psi)) \\
& =\mathbb{E}_{D \mu^{W}}\left[f_{\text {hor }}\right] \text {. }
\end{aligned}
$$

\section{Acknowledgements}

We would like to thank Christophe Sabot and Xiaolin Zeng for explaining their results and Letac's formula to us in different occasions. We would also like to thank Pierre Tarrès for sending us a copy of Letac's proof of his formula. 


\section{References}

M. Disertori, F. Merkl and S. W. W. Rolles. A comparison of a nonlinear sigma model with general pinning and pinning at one point. Electron. J. Probab. 21, Paper No. 27, 16 (2016). MR3485369.

M. Disertori and T. Spencer. Anderson localization for a supersymmetric sigma model. Comm. Math. Phys. 300 (3), 659-671 (2010). MR2736958.

M. Disertori, T. Spencer and M. R. Zirnbauer. Quasi-diffusion in a 3D supersymmetric hyperbolic sigma model. Comm. Math. Phys. 300 (2), 435-486 (2010). MR2728731.

G. Letac. The Sabot-Tarrès integral: proof and extensions (2014). Unpublished manuscript.

C. Sabot and P. Tarrès. Edge-reinforced random walk, vertex-reinforced jump process and the supersymmetric hyperbolic sigma model. J. Eur. Math. Soc. 17 (9), 2353-2378 (2015). MR3420510.

C. Sabot, P. Tarrès and X. Zeng. The vertex reinforced jump process and a random Schrödinger operator on finite graphs $(2017+)$. To appear in Ann. Probab.

C. Sabot and X. Zeng. A random Schrödinger operator associated with the vertex reinforced jump process on infinite graphs. ArXiv Mathematics e-prints (2015). arXiv: 1507.07944.

M. R. Zirnbauer. Fourier analysis on a hyperbolic supermanifold with constant curvature. Comm. Math. Phys. 141 (3), 503-522 (1991). MR1134935. 